\title{
Traditional Medicinal Plants Conferring Protection Against Ovalbumin-Induced Asthma in Experimental Animals: A Review
}

\author{
Shazalyana Azman ${ }^{1,2, *}$ \\ Mahendran Sekar (D) ${ }^{1, *}$ \\ Srinivasa Reddy Bonam (DD ${ }^{3, *}$ \\ Siew Hua Gan (1D ${ }^{4}$ \\ Suzana Wahidin ${ }^{2}$ \\ Pei Teng Lum' \\ Shivsharan B Dhadde ${ }^{5}$ \\ 'Department of Pharmaceutical \\ Chemistry, Faculty of Pharmacy and \\ Health Sciences, Universiti Kuala Lumpur \\ Royal College of Medicine Perak, Ipoh, \\ Perak, 30450, Malaysia; ${ }^{2}$ Bioengineering \\ and Technology Section, Universiti Kuala \\ Lumpur Malaysian Institute of Chemical \& \\ Bioengineering Technology, Alor Gajah, \\ Melaka, 78000, Malaysia; ${ }^{3}$ Institut \\ National de la Santé et de la Recherche \\ Médicale, Centre de Recherche des \\ Cordeliers, Equipe-Immunopathologie et \\ Immunointervention Thérapeutique, \\ Sorbonne Université, Université De \\ Paris, Paris, France; ${ }^{4}$ School of Pharmacy, \\ Monash University Malaysia, Bandar \\ Sunway Selangor Darul Ehsan, 47500, \\ Malaysia; ${ }^{5}$ D.S.T.S. Mandal's College of \\ Pharmacy, Solapur, India
}

*These authors contributed equally to this work

\begin{abstract}
Asthma is a chronic inflammatory disease of the respiratory tract in which the numerous immune cells, including eosinophils, neutrophils, macrophages, T-lymphocytes, mast cells and epithelial lining play key roles. The numerous anti-asthmatic drugs are available in modern medicine to treat asthma, but they have several disadvantages, including side effects and the cost variations, which compromise treatment compliance. The literature review reveals that traditional herbal medicines have good potential as alternative treatment and management for asthma. However, communities hesitated to use the traditional herbal medicines due to lack of established mechanism of action about their anti-asthmatic potential. The present review aimed to summarise the information stated in the literature about the potential effect of traditional medicinal plants (TMPs) conferring protection against ovalbumin (OVA)-induced asthma model. The literature search was conducted in database like PubMed, Scopus, Google Scholar and ScienceDirect. After screening through the literature from 2011 to date, a total of 27 medicinal plants and two polyherbal extracts have been reported to be used as traditional herbal medicines and also utilised to be tested against OVAinduced asthma, were included. We found them to be an important alternative source of treatment for asthma, since some have comparable efficacies with drugs commonly used in the modern system against asthma. All the reported medicinal plants confirmed their traditional use against asthma or its related inflammation. The present review provides faith in traditional information and also offers new insight into the potential of natural products against asthma.
\end{abstract}

Keywords: OVA-induced asthma, traditional medicinal plants, natural products, allergic asthma, inflammation, T-helper cells

\section{Introduction}

Asthma, one of the chronic inflammatory airway diseases, which affecting 300 million people worldwide and is expected to be nearly 400 million by the next 5 years. The incidence of asthma is high and accounting for 1 out of 250 deaths worldwide. ${ }^{1}$ Majority of the asthma treatment (as recommended by the Global Initiative for Asthma), target to reduce the symptoms via ameliorating the inflammatory processes. ${ }^{2,3}$ To date, current asthma therapies are mainly based on pulmonary, followed by oral or intravenous administrations. The drugs of choice include beta-adrenoceptor-2 ( $\beta 2)$ agonists, corticosteroids as well as xanthines and their derivatives. For symptomatic relief from asthma $\beta 2$ agonists are often the drugs of choice. ${ }^{3,4}$ Nevertheless, given the wide choices of available anti-asthmatic 
drugs, they produce numerous side effects, comprising headaches, nausea and convulsions (xanthines), ${ }^{5}$ cardiovascular effects ( $\beta 2$ agonists), ${ }^{6}$ vomiting (phosphodiesterase type 4 inhibitors), ${ }^{7-9}$ osteoporosis, myopathies, adrenal suppression and metabolic disturbances, which may compromise the patients growth especially in young children (corticosteroids). $5,7,10,11$

Traditional medicine encompasses the total skill, knowledge and practices used in the maintenance of health as well as in the prevention, diagnosis, enhancement, or treatment of physical and mental illness which can be based on myths, perceptions, and experiences indigenous to various cultures. ${ }^{12}$ The use of traditional medicinal plants (TMPs) as herbal medicines which have good potential for treating physiological disorders like asthma or as an adjuvant to modern therapy has been extensively reported since ancient times. In addition, ethnopharmacological studies have been considered as an important tool for bioprospecting new bioactive compounds from medicinal plants. ${ }^{13}$ Although significant progress has been achieved in the synthetic chemistry that involves the synthesis of new molecules, drugs or active constituents developed from medicinal plants are until preserved. ${ }^{14,15}$

Traditional herbal medicine is often referred as "complementary and alternative medicine" which includes traditional Chinese medicine, traditional Indian medicine (Ayurveda), traditional Japanese medicine (Kampo) and traditional Korean medicine which are the most widely used systems today. Since all these traditional systems utilise medicinal plants and have been practiced for hundreds or even thousands of years all over the world, some have morphed into orderly regulated medicine systems. ${ }^{16-18}$ Nevertheless, to date, there is a lack of information on the use of TMPs as well as their experimental evidences.

Rural individuals from developing countries tend to have more trust in traditional herbal medicine due to their considerable experience and lack of access to modern medicines. Hence, in-depth information on traditional and ethnomedical perspectives can facilitate communitycentered approaches under the present medical system given that the potential of traditional herbal medicines in the treatment of asthma is not fully utilized. Thus, established preclinical studies are needed. Several experimental animal models for asthma are accessible for preclinical screening. Among them, OVA-induced asthma animal model has been widely used.
OVA is the main protein found in egg white, which is not intrinsically immunogenic and therefore have to be injected into the systemic circulation in the presence of adjuvants (substances that increase the immunogenicity of an antigen) typically aluminium hydroxide (Alum). ${ }^{19}$ Immunization of OVA along with alum in animals induces $\mathrm{T}$ helper (Th) cell (preferably Th2) responses, ie, called sensitisation. Further, immunization of OVA to the sensitized animals synergize the former immune responses, ie, called challenge. Subsequently, challenged animal's exhibit many of the features seen in asthmatic individuals including inflammation, infiltration of eosinophils, production of Th2 cytokines, increase in serum IgE and airway hyper-responsiveness (AHR). OVA challenge models have been long-established to be worthwhile for the preclinical assessment of potential therapeutic agents for asthma, where specific questions regarding allergic asthma can be addressed.

To gain insight into the potential role of natural products, an overview of various traditionally used medicinal plants against asthma and OVA-induced asthma models has been represented here. The attempt has been made to outline the summary of information gathering the anti-asthmatic potential of medicinal plants against OVAinduced asthma and correlated with their traditional use was performed. This review provides a significant contribution to bridging these evidence gaps, creating a resource for safe and effective implementation of TMPs among the communities, confirming their pharmacological activities, potential efficacies and safety as an alternative medicine for asthma treatment.

\section{Methods}

In this review, relevant studies were collected from several scientific databases, including PubMed, Scopus, Google Scholar and ScienceDirect (between 2011 and to date). The keywords used for the search include "Traditional herbal medicine" OR "Traditional Indian medicine" OR "Ayurveda" OR "Traditional Chinese medicine" OR "Traditional Korean medicine" OR "Traditional Japanese Medicine" OR "Kampo" OR "Herbal medicine" OR "Natural products" OR "Medicinal plants" OR "Plant extract" AND "Asthma" AND "Ovalbumin" AND "OVA" AND "OVA-induced allergic asthma" AND Anti-asthmatic AND "OVA-induced lung inflammation". Studies apart from English language and those that does not have full article were excluded from this review. The medicinal plants studied for OVA-induced asthma model, but without 
supporting any traditional use related to the management and treatment of asthma has been omitted. After applying the inclusion and exclusion criteria's and eliminating the duplicates from one to another database, a total of 27 TMPs and two polyherbal extracts investigated against OVA-induced asthma model along with its supportive evidence on its traditional use against asthma or its related inflammation were included in the present review.

\section{Anti-Asthmatic Potential of TMPs Against OVA-Induced Asthma in Animals}

\section{Animal Model of OVA-Induced Asthma}

The use of an appropriate animal model of allergy can help unravel unanswered questions in human allergic diseases. ${ }^{20-23}$ Therefore, the following ideal characteristics should be considered for any allergic asthma animal model.

- Induced allergic parameters, both clinical and immunologic reactions, should mimic humans.

- Animal model of allergy-induced asthma by protein antigen alone is ideal, rather than in combination with adjuvants.

- The animal model should be less complicated, with a limited number of sensitisations.

- An ideal animal model serves as a powerful tool for understanding the immunopathogenic mechanisms of the various drug candidates.

Among the different species, mouse models are preferred in allergen-induced studies when compared to rat models. ${ }^{24}$ Although various other mouse models (C57BL/ 6 and $\mathrm{A} / \mathrm{J}$ strains) are available, interestingly, BALB/c mice model, which have a high tendency of developing allergic responses, has been widely used. ${ }^{25}$ The foremost reason for the high allergic responses is due to the Th2 phenotype where BALB/c mice easily develop Th2-biased immune response. In addition, BALB/c successfully develops clinical symptoms, which are relatable to humans, including infiltration of leukocytes, airway inflammation and wall remodeling (goblet cell hyperplasia or metaplasia, epithelial hypertrophy, epithelial basement membrane thickening), mucus secretion, total and allergen-specific serum IgG1 and IgE, cytokine profile, among others. ${ }^{26}$ As an example, BALB/c mice sensitised with the insect allergen (Forcipomyia taiwana [biting midge]) show a similar type of eosinophil and lymphocyte infiltrations observed in humans. ${ }^{27}$ Furthermore, BALB/c mice model can also be used for exploration of novel mechanisms independent of cytokines (IL-4 and IL-5) in allergic airway hyperreactivity. ${ }^{28}$ In addition, many studies have confirmed the utility of $\mathrm{BALB} / \mathrm{c}$ mice as a model for allergy. $^{24,29-31}$ Having said that induction of allergenspecific hypersensitivity reactions are also species- and strain-dependent, ${ }^{31,32}$ indicating that a balance of these factors are required when selecting the best model.

\section{Pathophysiology of OVA-Induced Asthma}

Despite different pathophysiological mechanisms in allergen-induced asthma, OVA-induced classical eosinophilic asthma is common. Moreover, OVA in combination with endotoxin (lipopolysaccharide, LPS) at different sensitisation and challenge schedules were used to induce neutrophilic and mixed-granulocytic asthma. ${ }^{23,33}$ Although OVA at high doses is sufficient to induce asthma, the use of endotoxin and/or adjuvant can help exacerbate the OVAinduced immune response. For this, several sensitisation and challenge (acute or chronic) protocols have been developed and used. The use of the endotoxin (LPS) and adjuvant (Alum) may overcome the tolerogenic issues of OVA. Moreover, the use of endotoxin in the protocols are preferred in studies focusing on acute neutrophilia. Similarly, an adjuvant such as Alum, in particular, can be used to induce acute asthma models. Of note, Alum induces Th2-biased immune responses, thus synergising and facilitating OVA-induced immune response (Figure 1). Nevertheless, the inclusion of an adjuvant is not necessary for some type of allergy models such as food-induced allergy models.

In summary, OVA-induced asthma animal models have been used for 1) classification of allergen types, 2) assessing the efficacy of novel vaccine adjuvants, 3) understanding the molecular mechanisms of different types of allergies, and 4) evaluating potential drug candidates. All the above categories are based on pathophysiological observation, including allergen-specific $\operatorname{IgE}$ estimation, anaphylactic symptoms, blood histamine levels, immunophenotyping of mast cells and other subtypes, splenocyte cytokine profiles and histology.

\section{Selective TMPs Conferring Protection Against OVA-Induced Asthma}

All the medicinal plants reported in this review have been traditionally used to treat asthma or its related 


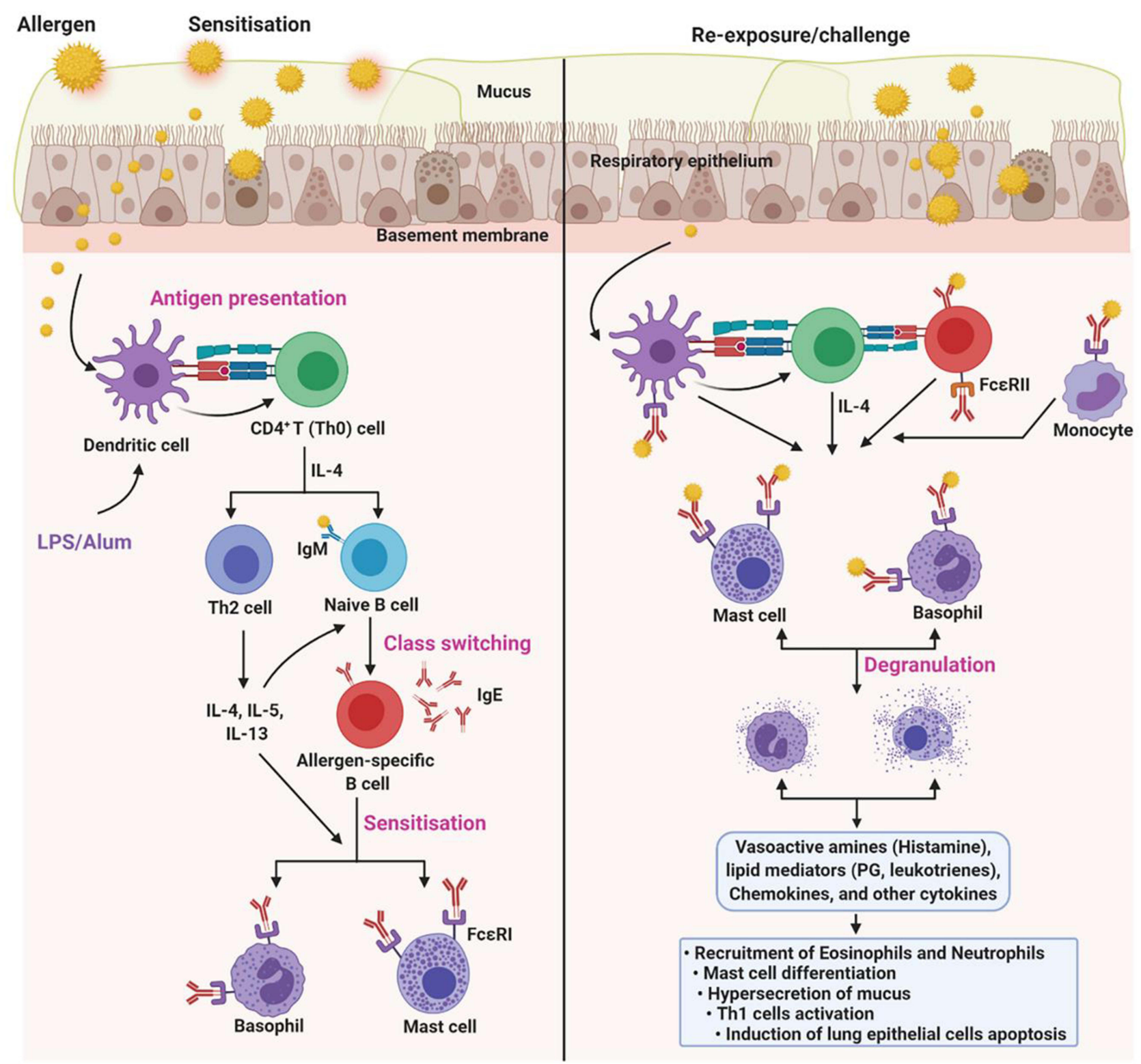

Figure I Pathophysiology of OVA-induced asthma. OVA-induces different types of allergic asthma, including eosinophilic, neutrophilic, and mixed-granulocytic asthma. Although different sensitisation and challenge protocols has been used, the underlying mechanism is nearly similar. Dendritic cells (DCs) are the first innate immune cells encounters the allergen after crossing the respiratory epithelium. DCs process and present the antigen to the CD4 ${ }^{+} \mathrm{T}$ cells, which subsequently polarized into Th2 cells via Th2 inducing factor (IL-4). Th2 cells secrete copious amounts of cytokines (IL-4, IL-5, and IL-I3), which later activate the naïve B cells to antigen-specific B cells (class switching). Further, antigen-specific B cells secret IgE, which sequentially recognized by the FcERI on the mast cells and basophils. This process is called sensitisation. Upon re-exposure, allergen experienced leukocytes accelerate the priming process and activate mast cells and basophils. In addition, sensitized mast cells and basophils directly recognize the lgE antigen conjugates and undergo degranulation, which releases vasoactive amines (histamine), lipid mediators (prostaglandin, leukotrienes), chemokines, and other cytokines. As a net effect, the former mediators recruit the eosinophils and neutrophils, induce the mast cell differentiation, hypersecretion of mucus, ThI cell activation, lung epithelial cells apoptosis, and others. ${ }^{137}$ Of note, few protocols use LPS or Alum, which accelerate the process, to induce a specific type of allergic condition. The figure was created with the support of https://biorender.com under the paid subscription.

inflammation. Table 1 summarises the details on the family, distribution, traditional uses and chemical constituents of TMPs and two polyherbal extracts. In this review, the data for 27 TMPs and two polyherbal extracts useful against OVA-induced asthma models as well as their potential mechanism of actions are described later.
The treatment with ethanolic extract of Scrophularia striata ( $S$. striata) to asthmatic mice shown suppressed Th2 cytokines (including IL-4 and IL-5) in bronchoalveolar lavage fluid (BALF) and inflammatory cells. In addition, it also decreased the level of total immunoglobulin E (IgE) and OVA-specific IgE in serum. ${ }^{34}$ These results make evident that $S$. striata extract have good potential in 
modulating Th2 cytokines and therefore, can be utilised as an immunomodulatory agent for allergic asthma. Almohawes and Alruhaimi ${ }^{35}$ reported that of hydroalcoholic extract of Lavandula dentata ( $L$. dentata) reduced the serum levels of IgE, triglycerides, total cholesterol and glucose in guinea pig with OVA-induced asthma model. Moreover, it also attenuated the levels of malondialdehyde (MDA) and restored the glutathione (GSH) levels in the lungs tissue. These results indicate that $L$. dentata extract ameliorates asthma and the oxidative stress as induced by OVA.

The protective effect of $50 \%$ ethanolic extract of Pistacia weinmannifolia (P. weinmannifolia) root (EEPWR) on inflammation and hypersecretion of mucus in respiratory tract in BALB/c mice was studied by Lee et al. ${ }^{36}$ Treatment with EEPWR decreased the eosinophil count and Th2 cytokines (IL-4, IL-5 and IL-13) levels in the BALF of OVA-exposed mice. Moreover, EEPWR inhibited total and OVA-specific IgE levels in serum. Additionally, EEPWR also efficiently inhibited the accumulation of inflammatory cells into the lungs, as well as respiratory tract mucus hypersecretion. Moreover, the monocyte chemoattractant protein-1 was significantly reduced following EEPWR treatment in the BALF of OVA-exposed mice. The protective effects of EEPWR on OVA-induced inflammation of respiratory tract were accompanied by the downregulation of mitogen associated protein kinases and activation of nuclear factor- $\kappa \mathrm{B}$. Overall, the studies indicate that EEPWR is a valuable armamentarium for asthma treatment. ${ }^{36}$

The anti-inflammatory and immunomodulatory activity of hydro-ethanolic extract of Ocimum basilicum (O. basilicum) leaves in OVA-challenged rats were examined by Eftekhar et al. ${ }^{37}$ Administration of $O$. basilicum extract decreased the levels of IL-4, IgE, phospholipase A2 and total protein and increased interferon- $\gamma($ IFN- $\gamma$ )/IL4 ratio in OVA-challenged rats. Additionally, O. basilicum extract showed improvements in OVA-induced changes in immunological, pathological and inflammatory markers in OVA-sensitised rats. The results were comparable and can even be more potent than that for standard dexamethasone, suggesting its therapeutic potential in asthma.

The anti-inflammatory actions of an ethanolic extract of Polyscias fruticosa ( $P$. fruticosa) leaves in OVAinduced asthma was tested by Koffuor et al. ${ }^{38}$ Administration of $P$. fruticosa extract reduced the OVAinduced elevation of white blood cells (WBC) and $\mathrm{C}$-reactive protein to normalcy. Moreover, administration of $P$. fruticosa extract reduced the rate of erythrocyte sedimentation. In the recent study, OVA-sensitized $\mathrm{BALB} / \mathrm{c}$ mice were treated with ethanolic extract of aerial parts of Herissantia tiubae (H. tiubae) 1 day before evaluating the anxiety and respiratory parameters. Increase in plasma IL-13, which was restored with the treatment of $H$. tiubae extract was observed. Additionally, there was a rise in total BALF cell count with prominent eosinophilia has been seen. It was determined that $H$. tiubae extract can show similar action on respiratory parameters to that of aminophylline and behavioral changes as that of diazepam. Suppression of inflammation was as efficient as that for dexamethasone. ${ }^{39}$

The effects of aqueous extract of Solanum xanthocarpum (S. xanthocarpum) on respiratory tract inflammation and oxidative stress in experimental asthmatic rats were assessed by Gulati. ${ }^{40}$ The administration of S. xanthocarpum extract $(50 \mathrm{mg} / \mathrm{kg})$, reduced the levels of blood and BALF OVA-specific IgE in Ova-immunised rats by $37 \%$ and $20 \%$, respectively. These effects were comparable with prednisolone $(10 \mathrm{mg} / \mathrm{kg})$, which reduced IgE level by $43 \%$ and $31 \%$ in blood and BALF respectively. Similarly, administration of $S$. xanthocarpum extract at the dose of $100 \mathrm{mg} / \mathrm{kg}$ attenuated the levels of tumor necrosis factor- $\alpha$ (TNF- $\alpha$ ), IL-6 and IL-4 and elevated the levels of IFN- $\gamma$ when compared with control. These data indicate improved immunostimulatory/immunomodulatory effects. In addition, administration of S. xanthocarpum extract $(100 \mathrm{mg} / \mathrm{kg})$ ameliorates oxidative stress in the animals and it is indicated by reduced MDA levels and elevated GSH levels. It was hypothesised that the beneficial effects of $S$. xanthocarpum in bronchial asthma are attributed to the balancing influence on prooxidant-antioxidant status and reduction in airway inflammation.

The effect of $70 \%$ ethanolic extract of Crocus sativus (C. sativus) flowers on differential and total WBC count in lung lavage fluid of Ova-sensitised rats were reported by Mahmoudabady et $\mathrm{al}^{41}$ C. sativus extract treatment decreased percentage of neutrophils and eosinophils and total WBC count and the in OVA-sensitised animals. Therefore, the findings indicated that $C$. sativus extract is effective in alleviating lung inflammatory cells in experimentally induced asthmatic animals. ${ }^{41}$ Bulani et $\mathrm{al}^{42}$ reported the effect of methanolic extract of Calotropis gigantea ( $C$. gigantea) root on OVA-induced asthma in rats. C. gigantea extract-treatment showed significant inhibition of neutrophil, lymphocyte, eosinophil and total 
leukocyte count in BALF of animals treated with OVA. Moreover, $C$. gigantea extract reduced the oxidative stress in OVA treated animals and it was evident by reduced nitric oxide (NO) levels in BALF and lipid peroxidation (LPO) in the lungs and restored levels of GSH, superoxide dismutase (SOD) in lungs. C. gigantea extract also significantly improved the OVA-induced histological changes. These results suggest the potential effect of C. gigantea in OVA-induced asthma.

The anti-asthmatic and antioxidant activities of aqueous extract of Urtica dioica (U. dioica) leaves in OVAsensitised rats were examined by Zemmouri et $\mathrm{al}^{43}$ The aqueous extract of $U$. dioica leaves significantly inhibited serum eosinophilia (by 60\%), leucocyte (by $32.75 \%$ ) and lymphocytes (by 29.22\%) in BALF of asthmatic rats. Besides, LPO generated following allergen administration was significantly diminished by $U$. dioica treatment in the lung tissues (by 48.58\%), thus confirming that $U$. dioica aqueous leaves extract confer some protective effects against airway inflammation. Khan and Imtiyaz ${ }^{44}$ reported the ethanolic extract of Wrightia tinctoria ( $W$. tinctoria) leaves treatment decreased leukocyte count when compared to OVA-induced animals. The findings indicate that ethanolic leaves extract of $W$. tinctoria possess some antiasthmatic effects, possibly mediated via inhibition of histamine release.

The administration of Asparagus cochinchinensis (A. cochinchinensis) root extract lowered the bronchial thickness and infiltration of inflammatory cells, also showed a reduction in macrophages, eosinophils, IgE and Th2 cytokines in OVA-induced animals. ${ }^{45}$ In addition, there was a significant recovery in goblet cell hyperplasia, matrix metallopeptidase-9 (MMP-9) expression and vascular endothelial growth factor (VEGF) signaling pathway upon airway remodelling in the extract-treated group. Overall, these findings provide strong evidence that A. cochinchinensis root extract prevents airway inflammation of a chronic asthma model. ${ }^{45} \mathrm{Sim}$ et a ${ }^{46}$ reported that $70 \%$ ethanolic extract of Aster yomena (A. yomena) decreased Th2 type cytokines and eosinophils in the BALF and OVA-specific IgE in serum. In addition to that, A. yomena extract reduced AHR and histopathology of lungs returned to normalcy in the OVA-challenged animals. ${ }^{46}$

The aqueous extract of Allium cepa (A. cepa) normalised the levels of nitrogen dioxide $\left(\mathrm{NO}_{2}\right)$, nitrate $\left(\mathrm{NO}_{3}\right)$, MDA, IL-4 and IgE, and the antioxidant markers such as SOD, catalase (CAT), thiol and IFN- $\gamma$ as well as the IFN- $\gamma /$
IL-4 ratio in OVA-sensitised animals. ${ }^{47}$ The OVA-induced animals when treated with Artemisia argyi (A. argyi), it has reduced inflammatory cells, cytokines and AHR in asthmatic animals. ${ }^{48}$ Furthermore, A. argyi decreased extracellular receptor kinase (ERK) phosphorylation as well as the expression of MMP-9 in asthmatic animal models, indicating its potential against allergic asthma. The aqueous and ethanolic extracts of rhizomes of Zingiber officinale ( $Z$. officinale) treated animals showed a reduction of total $\mathrm{WBC}$, eosinophils and neutrophils in BALF. $^{49}$ Moreover, Z. officinale extracts significantly inhibited Th2-mediated immune response, as evidenced by a decrease in mRNA expression levels of IL-4 and IL-5. Furthermore, there was a significant reduction in protein levels of IL-4 and IL-5 in BALF, as well as total serum IgE level by both extracts.

Seo et $\mathrm{al}^{50}$ reported that the $80 \%$ ethanolic extract of Erythronium japonicum (E. japonicum) treatment suppressed the number of $\mathrm{WBC}$ and decreased the IgE level in the BALF in mice treated with OVA. Furthermore, treatment with E. japonicum extract modulated the expression of both Th2 and Th1 cell-related factor. Trigonella foenumgraecum (T. foenum-graecum) treatment decreased Th2 cytokines and increased Th1 cytokines in BALF and lung homogenates. ${ }^{51}$ Furthermore, T. foenum-graecum significantly inhibited serum IgE and anti-OVA IgG1, indicated that it is a useful agent against allergic asthma. ${ }^{51}$ Treatment of $75 \%$ hydroethanolic extract of Echinodorus scaber (E. scaber) leaves reduced total eosinophil, neutrophil, leukocyte and mononuclear cell counts in OVA-induced allergic asthma. ${ }^{52}$ Further, E. scaber extract ameliorated the increased in Th2 cytokines and IgE levels, which confirmed the utility of the leaves in treating allergic inflammation.

Treatment of Siphonochilus aethiopicus (S. aethiopicus) to OVA-induced mice reduced allergic lung inflammation and eosinophils percentage in BALF, although it did not influence airway hyper-reactivity. ${ }^{53} \mathrm{Yu}$ et al ${ }^{54}$ investigated the protective effects of total flavonoids from $70 \%$ ethanolic extract of Selaginella uncinata (S. uncinata) on airway hyper-responsiveness, cytokine release and bitter taste receptors (T2Rs) signalling, emphasising on inflammatory responses in OVA-induced asthma in rats.

Treatment with $S$. uncinata extract attenuated AHR and goblet cell hyperplasia. Additionally, serum levels of Th2related cytokines include IL-4, IL-5 and IL-13, total and OVA-specific IgE were reduced and IFN- $\gamma$ was improved in $S$. uncinata-treated rats. They have also reported that S. uncinata utilises its anti-inflammatory role via T2R10/ 
IP3R1/NFAT1 dependent signaling pathway. ${ }^{54}$ Rosa multiflora (R. multiflora) extract inhibited eosinophil accumulation in nasal lavage fluid (NALF), the goblet cells and nasal mucosa in the nasal epithelium and mast cells in the respiratory region of the nasal cavity. ${ }^{55}$ Furthermore, R. multiflora extract suppressed Th2-related cytokines in NALF, splenocytes and nasal-associated lymphoid tissue (NALT), whereas Th1-associated cytokine IL-12 was upregulated, indicating that $R$. multiflora has therapeutic potential in treating allergic rhinitis by moderating the associations between $\mathrm{Th} 1 / \mathrm{Th} 2$ responses.

Dussossoy et $\mathrm{al}^{56}$ found that Morinda citrifolia (M. citrifolia) fruit juice reduced the inflammation in OVA-sensitised animals with regards to the decreased number of inflammatory cells in lung lymphocytes, macrophages, neutrophils and eosinophils. In a study, n-hexane fraction of $70 \%$ Scutellaria baicalensis (S. baicalensis) ethanolic extract has significantly suppressed the production of Th2-mediated cytokines and increased Th1mediated cytokines against OVA-induced animals. ${ }^{57}$ The $70 \%$ aqueous ethanolic extract of Sceptridium ternatum (S. ternatum) treatment significantly reduced AHR, raised the ratio of Th1/Th2 and reduced Cyslt 1 mRNA level in a dose-dependent manner. ${ }^{58}$ Moreover, high-dose of S. ternatum had similar efficacy as the investigated standard drug (Montelukast) in a mouse asthma model and therefore is a good potential anti-asthmatic agent.

The hydro-ethanolic extract of Curcuma longa (C. longa) significantly decreased tracheal responsiveness as well as pathological lung features in OVA-sensitised rats. Additionally, the extract decreased tracheal responsiveness to methacholine. Moreover, C. longa significantly decreased interstitial fibrosis compared to OVA-sensitized group. The $C$. longa treated animals showed similar or even more than that conferred by standard dexamethasone at $250 \mu \mathrm{g} / \mathrm{kg}{ }^{59}$ The administration of Zanthoxylum bungeanum (Z. bungeanum) seed oil lessened airway remodelling and lung tissue injury and inhibited the infiltration of eosinophils and leukocytes into the airway by decreasing the expression levels of inflammatory chemokines and cytokines compared with OVA-treated animals. ${ }^{60}$ Moreover, Z. bungeanum seed oil also reduced the levels of inflammatory adhesion molecules and chemokine via downregulation of extracellular signal-regulated kinase and activation of c-JUN N-terminal kinase (JNK).

Soshiho-tang, also known as "Xiao-Chai-Hu-Tang" (Chinese) and "Sho-Saiko-to" (Japanese), is composed of seven herbs (Table 1). ${ }^{61}$ The administration of Soshiho- tang water extract suppressed eosinophil influx into BALF while decreasing the levels of Th2-type cytokines. ${ }^{62}$ Moreover, Soshiho-tang water extract exhibited a marked decrease in mucus hypersecretion, IgE levels while significantly induced the expression of HO-1 protein. Therefore, it was determined that Soshiho-tang water extract might be another useful armamentarium against allergic asthma. In one another study, the pentaherbs formula (PHF) comprised of five traditional Chinese herbal medicines (Table 1). The water extract of PHF showed significant suppression of pulmonary eosinophilia and asthma-related cytokines IL-4 and IL-33 in BALF T. ${ }^{63}$

\section{Overall Potential Mechanism of Action of TMPs Against OVA-Induced Asthma}

Due to the ethical and moral issues subjecting asthma patients to traditional herbal medicines which may exacerbate their conditions in clinical trials, the development of preclinical animal model is of great importance. OVAinduced asthma is one of the most studied allergic asthma model across the world. In this review, it was noteworthy to state that there are several variations of the model in which OVA-sensitization and OVA-challenge have been conducted via different delivery modes including repeated intraperitoneal, subcutaneous and intranasal or intratracheal administrations. The overview of the detailed protocol followed to investigate the effect of TMPs against OVA-induced asthma has been summarized in Figure 2 and Table 2. When allergen/OVA enter the lungs, antigen-presenting cells (dendritic cells) captures and present the processed antigen to the $\mathrm{CD} 4^{+}$ $\mathrm{T}$ cells. Activated T cells produce copious amounts of Th2biased cytokines (IL-4, IL5, and IL-13), which further activates the Th 2 cells and B cells. Sequentially activated B cells secrete IgE, which is a stimulating factor for mast cells to produce inflammatory mediators. Furthermore, T cell activates the other innate immune cells, including eosinophils which aggravate the inflammatory situation. TMPs which composed of heterogenous components inhibit the allergen/ OVA-induced asthma by inhibiting either the antigen presentation, cytokine secretion or the release of inflammatory mediators. The detailed mechanism of TMPs against OVAinduced asthma was shown in Figure 3.

\section{Challenges and Opportunities of TMPs for the Treatment of Asthma}

Since asthma is a chronic disease, conventional management for asthma generally requires institution of extensive 


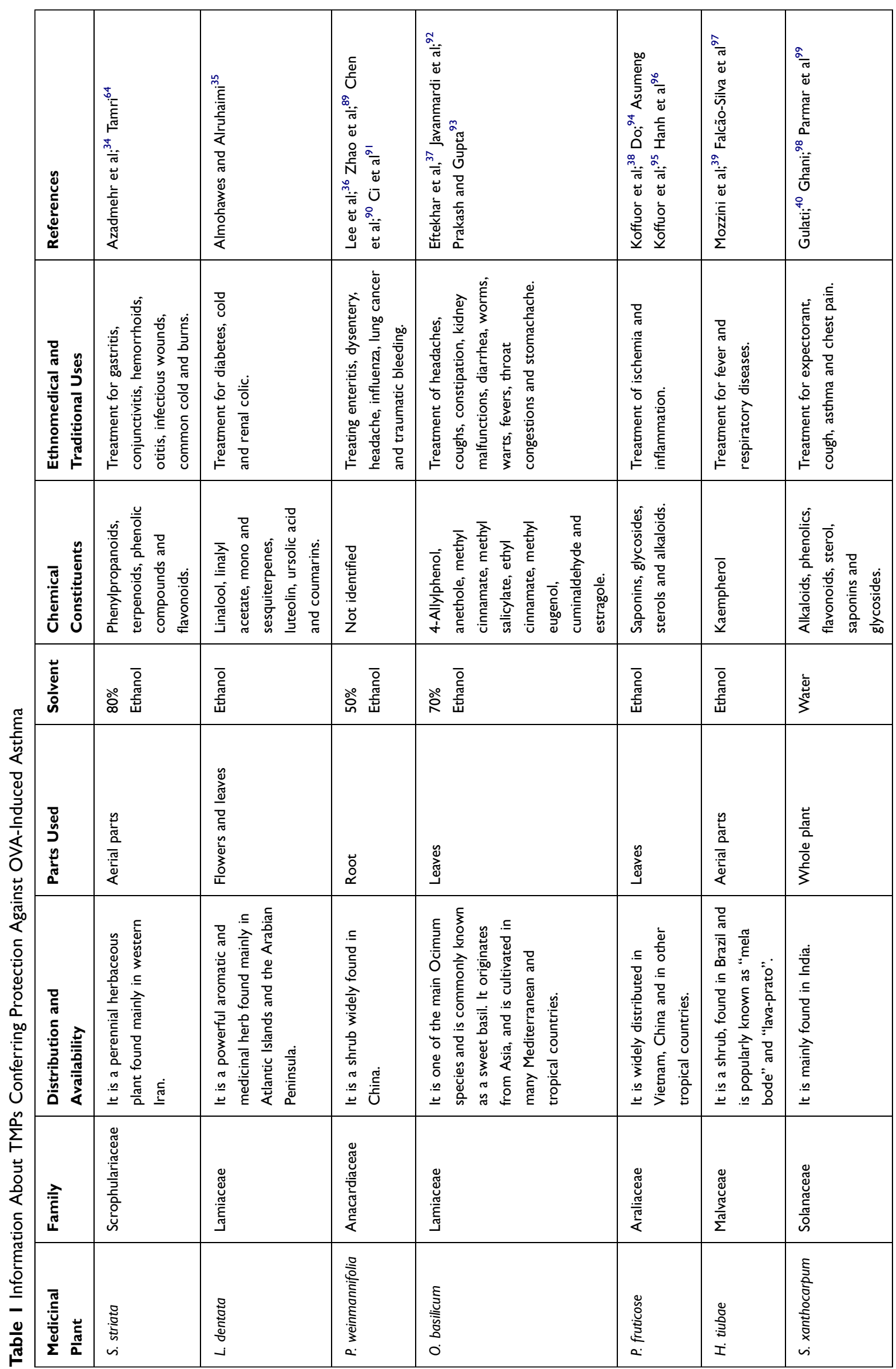




\begin{tabular}{|c|c|c|c|c|c|c|c|}
\hline 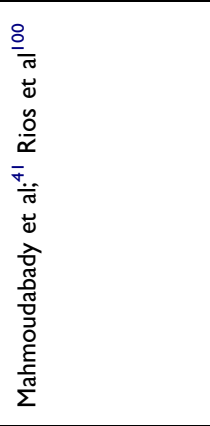 & 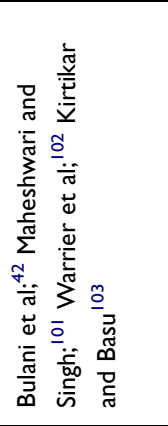 & 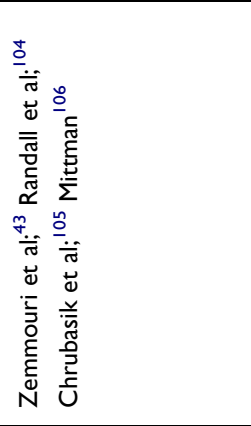 & 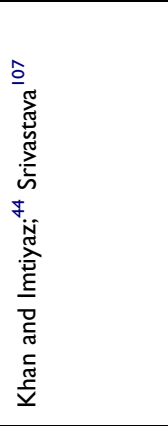 & 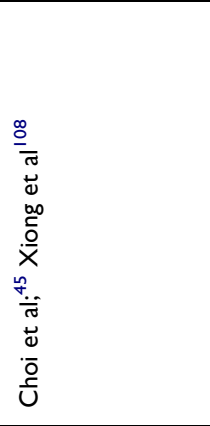 & 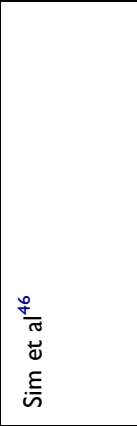 & 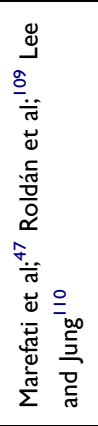 & 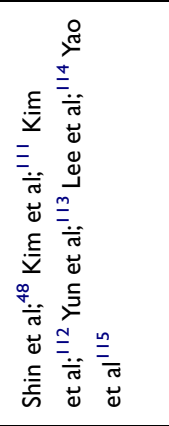 \\
\hline 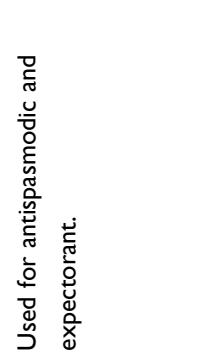 & 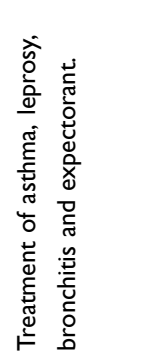 & 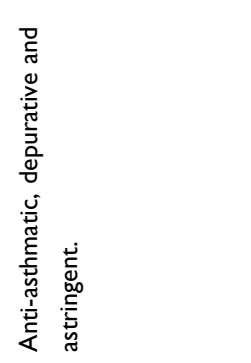 & 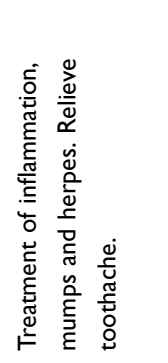 & 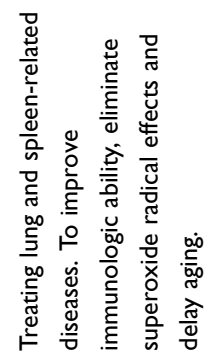 & 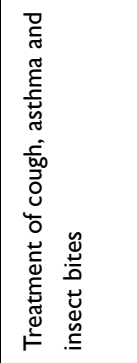 & 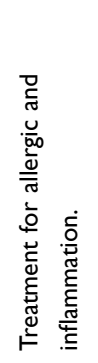 & 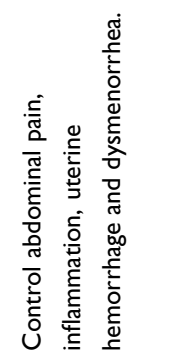 \\
\hline 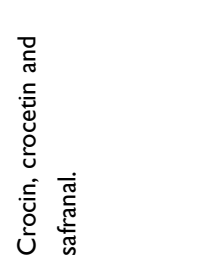 & 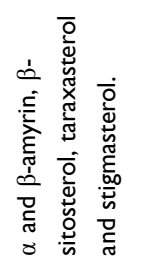 & 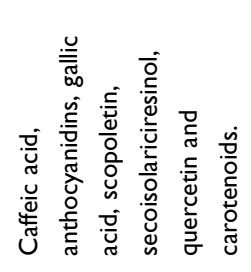 & 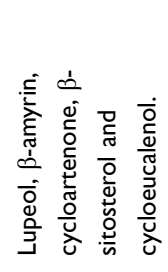 & 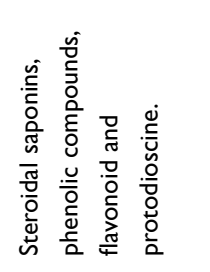 & 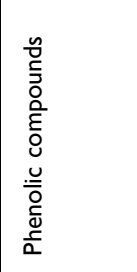 & 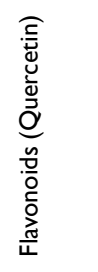 & 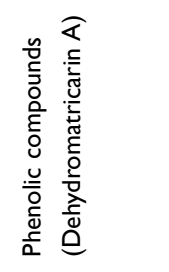 \\
\hline 号 & 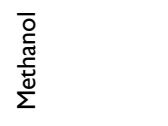 & $\begin{array}{l}\bar{\Phi} \\
\tilde{J}^{ \pm}\end{array}$ & 今。 & 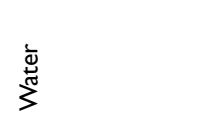 & ஓे & 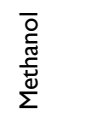 & $\begin{array}{l}\bar{o} \\
\bar{c} \\
\overline{\underline{c}} \\
\overline{\tilde{\omega}} \\
\Sigma\end{array}$ \\
\hline 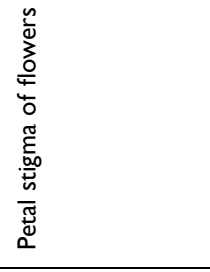 & $\begin{array}{l}\stackrel{\circ}{\circ} \\
\stackrel{\alpha}{\alpha}\end{array}$ & 岕 & 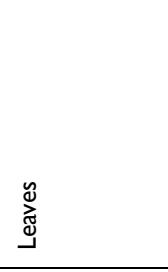 & 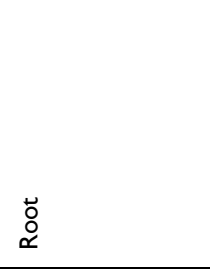 & 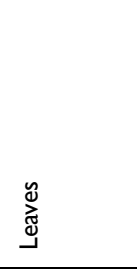 & 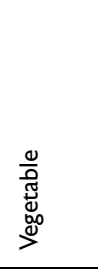 & 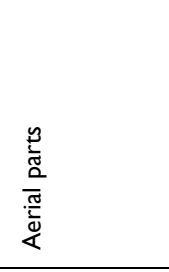 \\
\hline 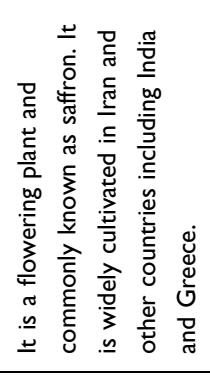 & 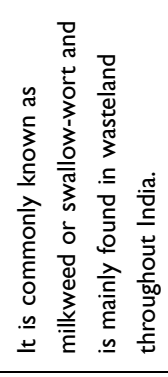 & 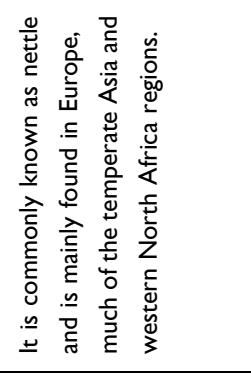 & 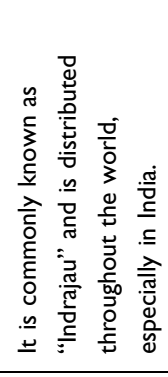 & 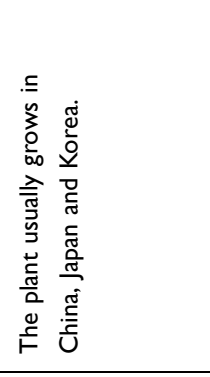 & 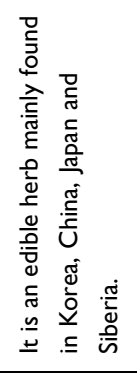 & 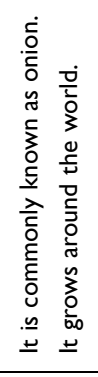 & 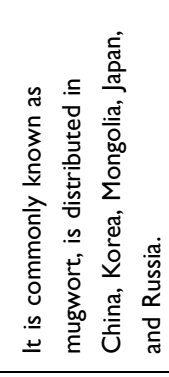 \\
\hline 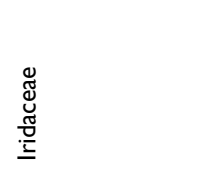 & 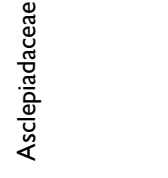 & 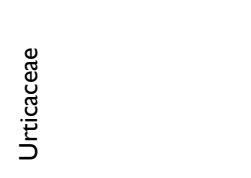 & 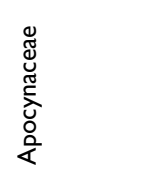 & 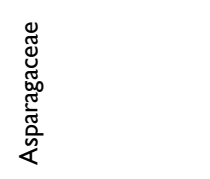 & 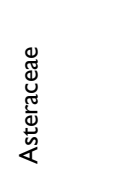 & 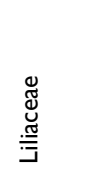 & 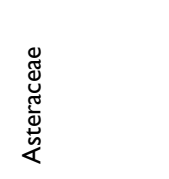 \\
\hline 逽 & 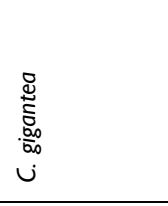 & $\begin{array}{l}\stackrel{g}{\circ} \\
\stackrel{0}{0} \\
د\end{array}$ & 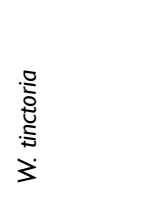 & 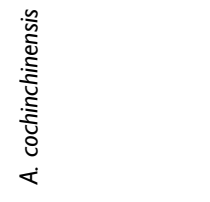 & 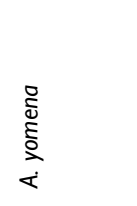 & 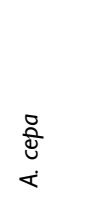 & 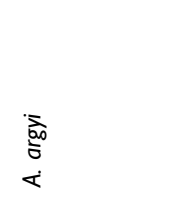 \\
\hline
\end{tabular}




\begin{tabular}{|c|c|c|c|c|c|c|}
\hline 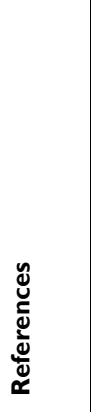 & 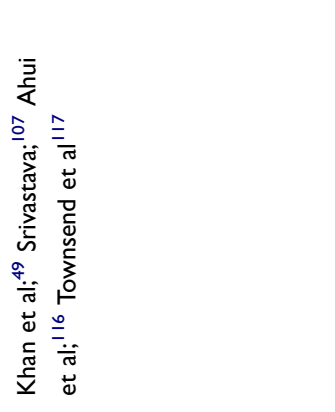 & 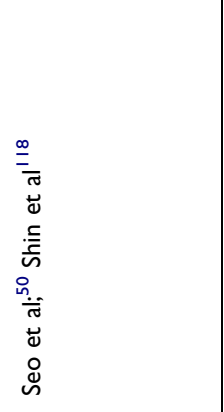 & 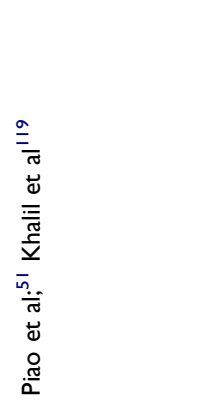 & 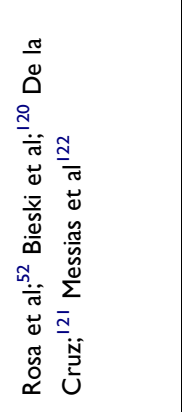 & 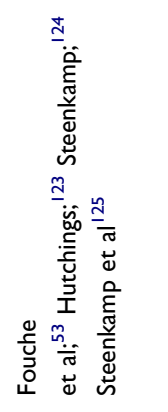 & 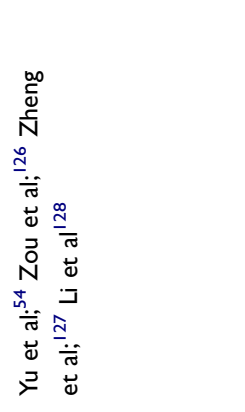 \\
\hline 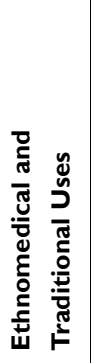 & 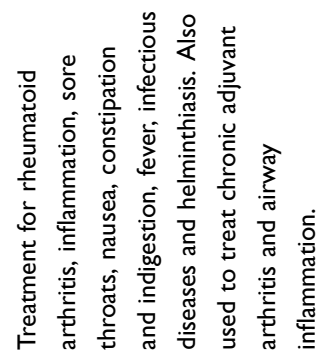 & 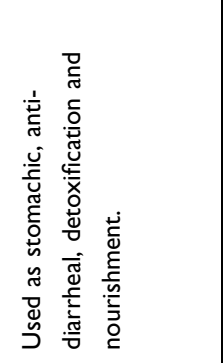 & 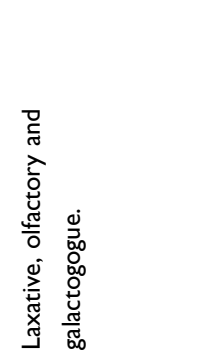 & 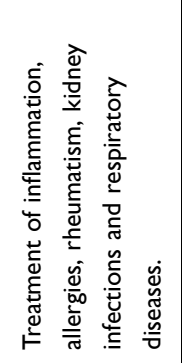 & 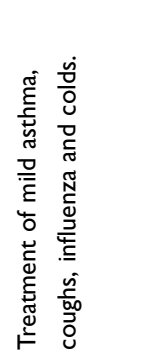 & 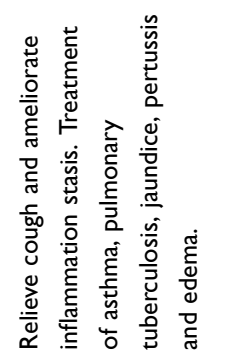 \\
\hline 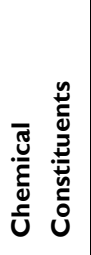 & 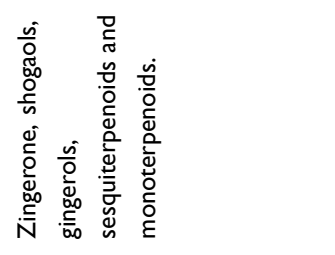 & 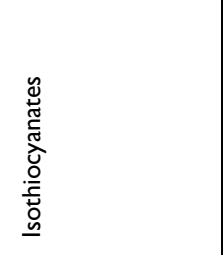 & $\begin{array}{l}\frac{n}{0} \\
\overline{0} \\
\frac{0}{0} \\
\frac{\pi}{L}\end{array}$ & 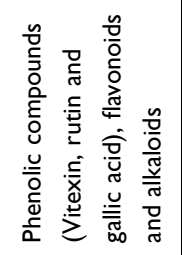 & 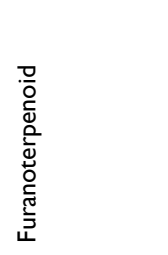 & 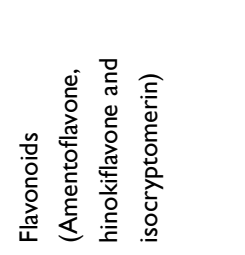 \\
\hline 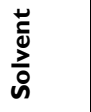 & 高 & 品 & $\begin{array}{l}\bar{o} \\
\text { 胥 } \\
\text { 壱 }\end{array}$ & 总 & 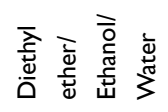 & 高 \\
\hline 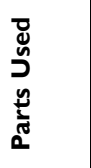 & $\begin{array}{l}\stackrel{0}{0} \\
\stackrel{0}{0} \\
\stackrel{N}{\bar{c}} \\
\frac{\alpha}{\alpha}\end{array}$ & 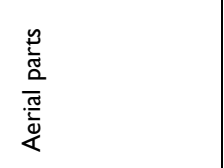 & 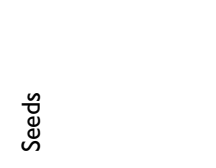 & 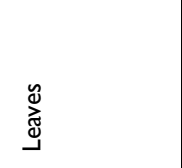 & 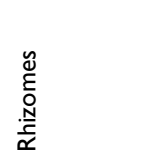 & $\begin{array}{l}\frac{\breve{c}}{\frac{\pi}{\alpha}} \\
\frac{0}{0} \\
\frac{0}{3}\end{array}$ \\
\hline 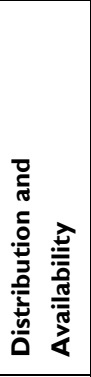 & 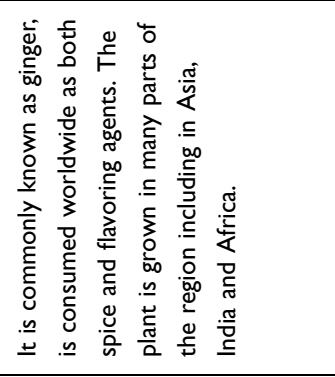 & 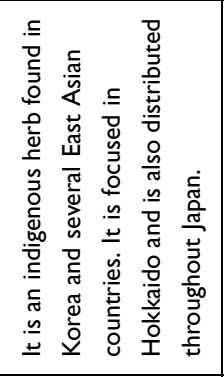 & 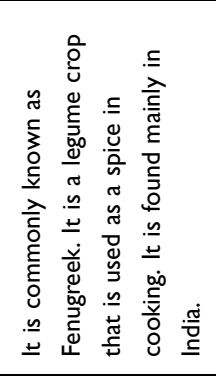 & 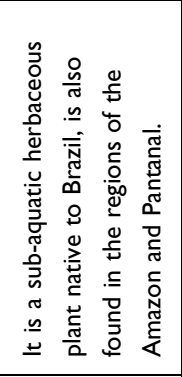 & 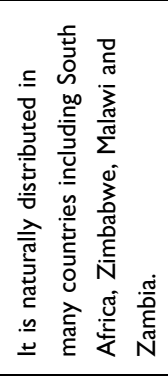 & 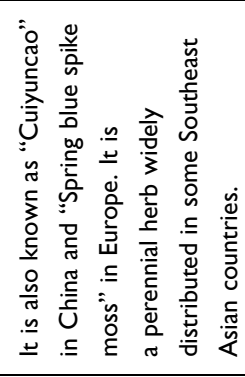 \\
\hline 츨 & 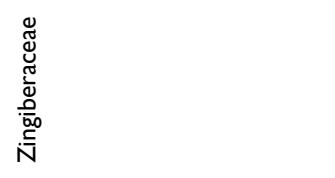 & 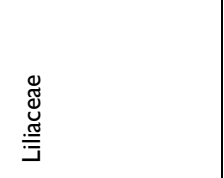 & 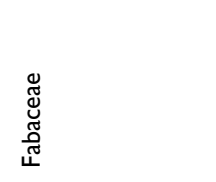 & 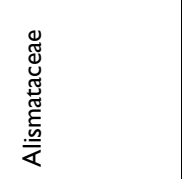 & 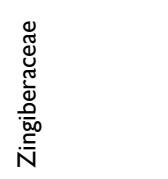 & 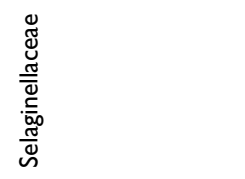 \\
\hline 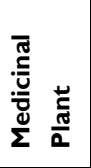 & 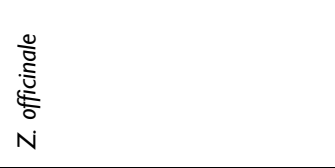 & 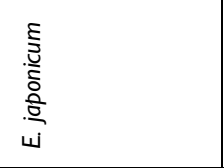 & 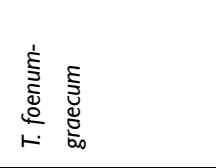 & 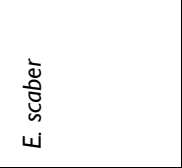 & 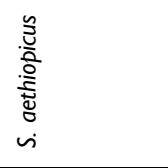 & 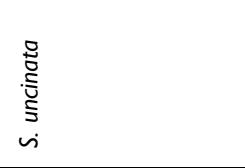 \\
\hline
\end{tabular}




\begin{tabular}{|c|c|c|c|c|c|c|}
\hline 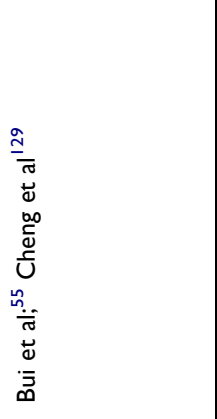 & 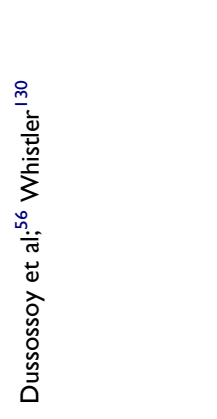 & 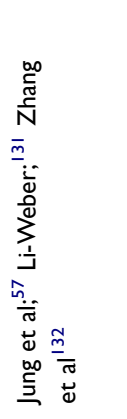 & 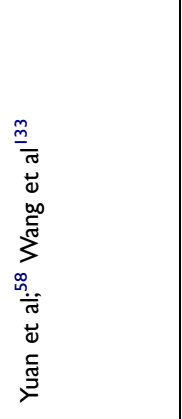 & 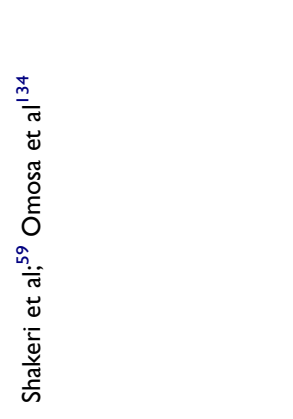 & 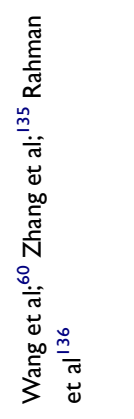 & \\
\hline 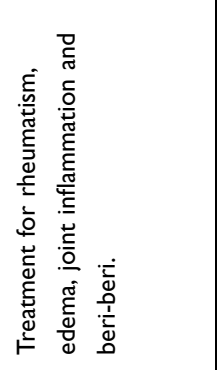 & 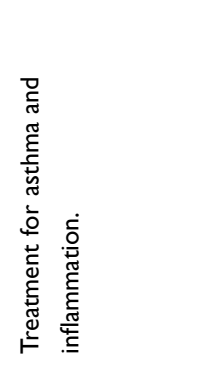 & 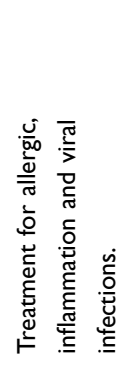 & 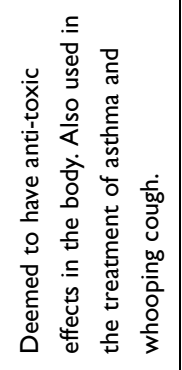 & 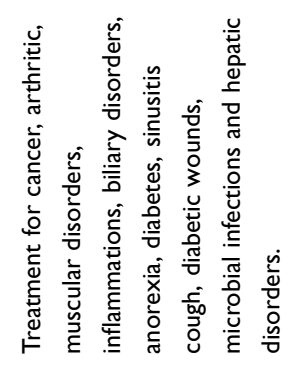 & 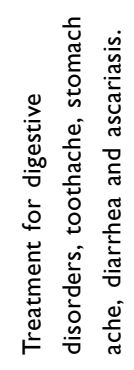 & \\
\hline 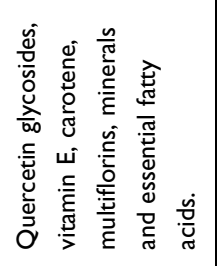 & 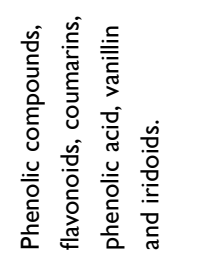 & 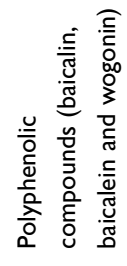 & 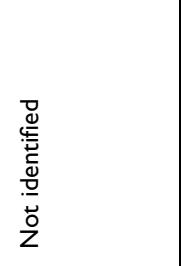 & 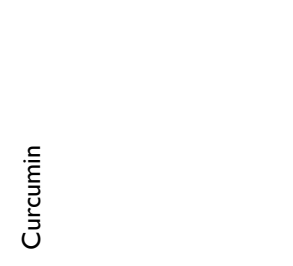 & 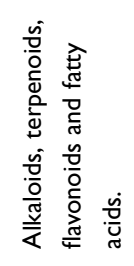 & \\
\hline 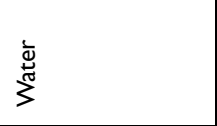 & 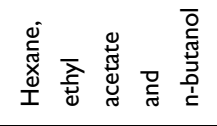 & 高 & 仓ำ & $\begin{array}{l}\bar{o} \\
\text { o } \\
\text { 胥 }\end{array}$ & 1 & \\
\hline$\stackrel{n}{\stackrel{n}{\underline{E}}}$ & 旁 & 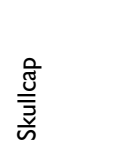 & 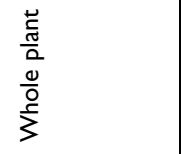 & 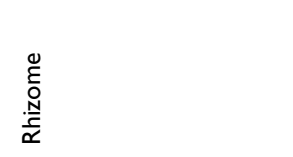 & 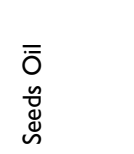 & \\
\hline 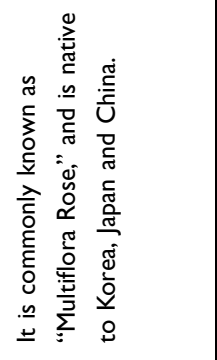 & 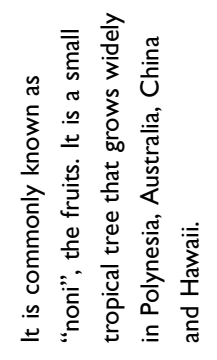 & 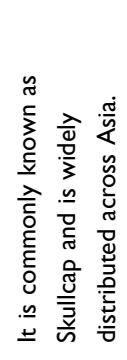 & 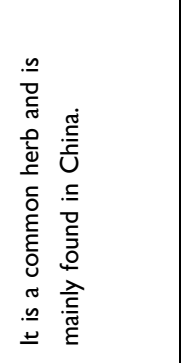 & 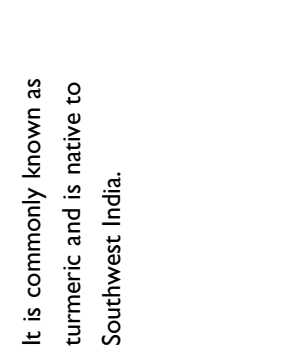 & 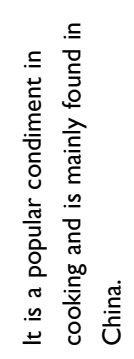 & \\
\hline 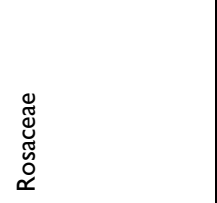 & 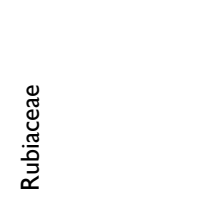 & 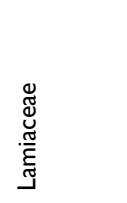 & 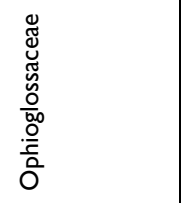 & 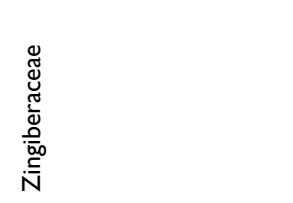 & 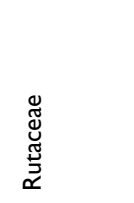 & \\
\hline 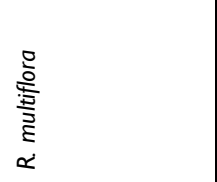 & $\begin{array}{l}\frac{0}{\frac{0}{0}} \\
\frac{5}{5} \\
\sum\end{array}$ & 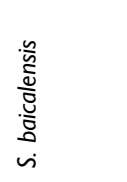 & 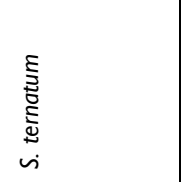 & 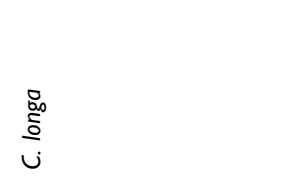 & 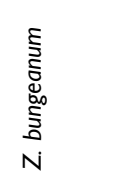 & 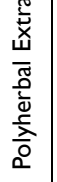 \\
\hline
\end{tabular}




\begin{tabular}{|c|c|c|}
\hline 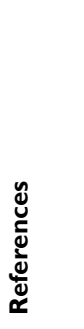 & 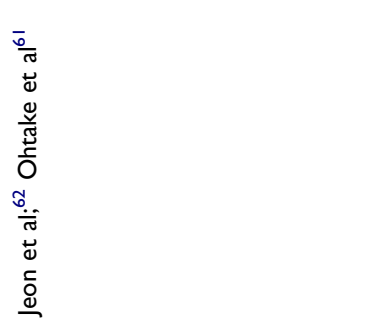 & 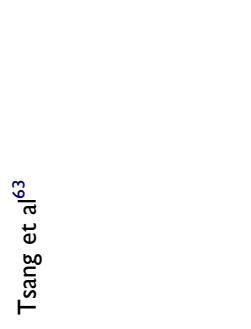 \\
\hline 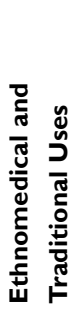 & 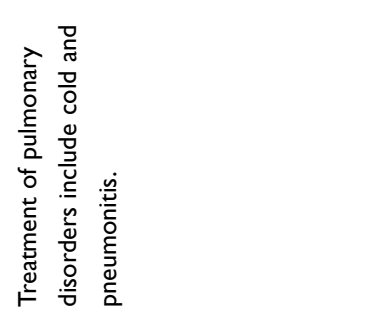 & 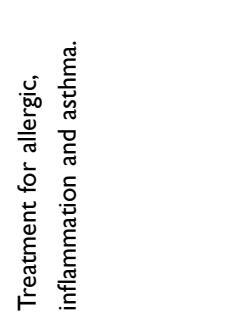 \\
\hline 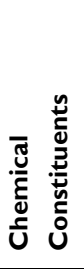 & 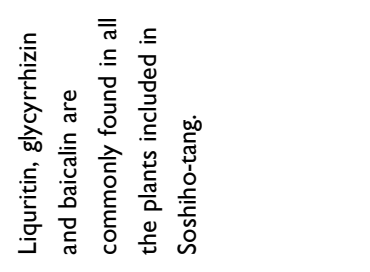 & 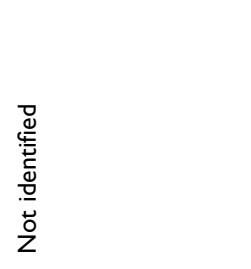 \\
\hline 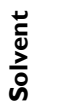 & 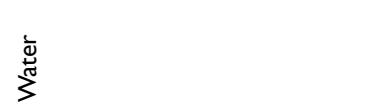 & 产 \\
\hline 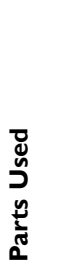 & 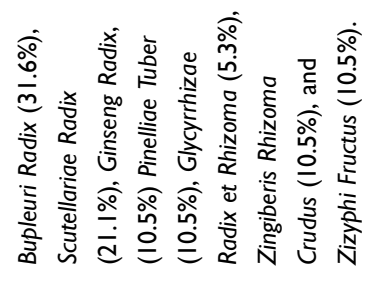 & 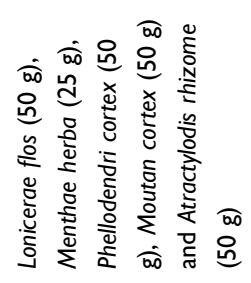 \\
\hline 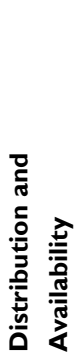 & 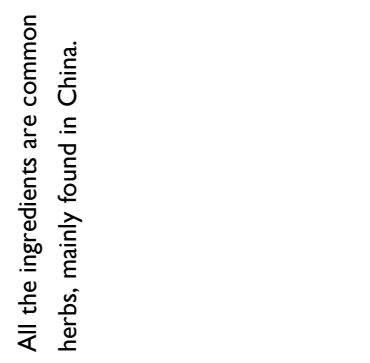 & 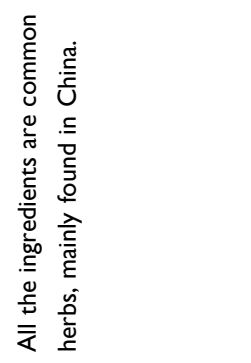 \\
\hline 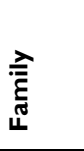 & 1 & 1 \\
\hline 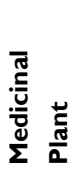 & 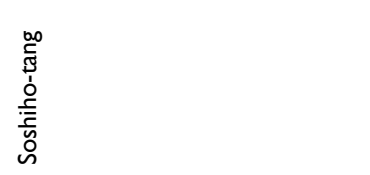 & 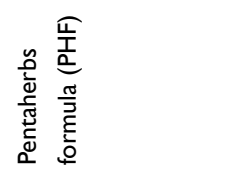 \\
\hline
\end{tabular}

bronchodilators and corticosteroids treatment. Nevertheless, drugs, especially steroids, are allied with various side effects and refractory responses. Therefore, TMPs may provide a better alternative strategy against asthma. Nevertheless, scientific validation remains lacking and is urgently required to confirm their utility either as a complementary alternative therapy or as an adjunct to conventional therapies. ${ }^{64}$

Based on the established literatures, administration of the reported medicinal plant extracts to OVA-induced animals can effectively inhibit the entry of inflammatory cells into the lungs, where a significant reduction in the total eosinophil and leucocyte populations in the lung tissue can be seen. All the medicinal plants stated in this review are used to treat respiratory inflammation, asthma or its related diseases for many years based on the traditional Indian medicine (Ayurveda), traditional Chinese medicine and other indigenous medicinal systems. The scientific reports reviewed have demonstrated that all these medicinal plants have potential anti-asthmatic effect against OVA-induced asthma model, which is similar to asthma experienced by humans which strongly support their traditional uses. However, further studies are required since most are established in the earlier phases of study and clinical trials are encouraged. Additionally, the safety, toxicity and side effect data associated with TMPs are severely lacking and have to be addressed. TMPs have several chemical constituents which are responsible for the management of asthma or its relevant complications. Thus, the exact active constituent responsible for its anti-asthmatic effects should be evaluated. Furthermore, its pharmacokinetic, pharmacological and clinical data has to be investigated to confirm its potential as a lead compound against asthma.

Moreover, an archive of information on herbal medicine from various cultural backgrounds (Indian, Chinese, Japanese, Korean, African and Arabian) for the management and treatment of asthma. ${ }^{65-81}$ To date, knowledge on the medicinal plants used for asthma medication and their mode of preparations are limited to some herbal medicine practitioners although some clinical and pharmacological evidences are available. ${ }^{68}$ Therefore, there is insufficient evidence to make recommendations to use of these TMPs in clinical practice since the global acceptance and utilisation of herbal medicines is dependent on their safety and efficacy.

Our review suggests that OVA-induced animal model may be used to confirm the effectiveness of the TMPs. The model confirms the effectiveness of herbal 


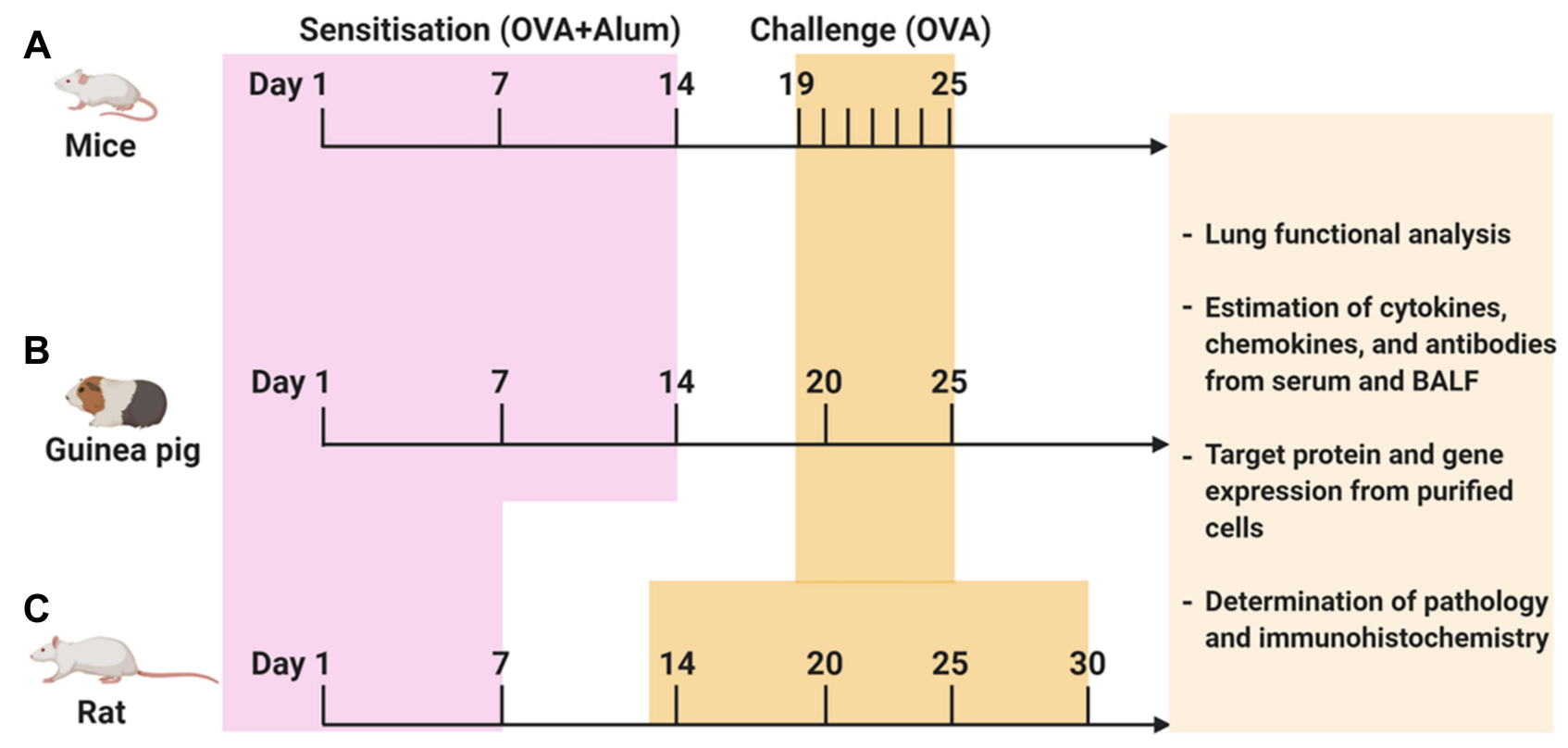

Figure 2 Protocol followed to investigate TMPs against OVA-induced asthma. OVA-induced animal asthma model: (A) mice, (B) Guinea pig, (C) Rat. The above figure depicts the timeline of OVA sensitization and challenge followed by the end readouts. Schedules of sensitization and challenge depend on the type of animal model and routes of administration. In addition, varying OVA doses (with or without adjuvant) have been used both in sensitization and challenge (for more information, see Table I).

medicines on typical inflammatory processes as seen in asthma inflammation which confirms the clinical biological plausibility of herbal medicines effects. Several new animal models are currently available for preclinical screening to confirm the efficacy of the antiasthmatic drugs. Thakur et al ${ }^{82}$ developed a new model to confirm the anti-asthmatic effects in which the combination of OVA with LPS demonstrated the phenotypes of severe lymphocytic inflammation, neutrophilic, eosinophilic and bronchoconstriction, when compared to a single allergen. Few other studies have also shown that pulmonary neutrophilia in mouse is triggered by the combination of LPS with commercial OVA. ${ }^{83,84}$ These new models can be exploited to evaluate the severe antiasthmatic effect of TMPs.

Very recently, several clinical studies have reported the use of TMPs for asthma treatment. Dulla and Jahan ${ }^{70}$ indicated that some traditional Chinese medicines indicated their effectiveness against asthma in children when compared to modern medicines such as salbutamol and montelukast. In another research, some of the traditional herbal medicines in East-Asia were used as adjuncts in improving acute and chronic asthma outcomes ${ }^{85}$ which boosts the evaluation of TMPs for clinical trials. Wang et $\mathrm{al}^{86}$ reported that the clinical efficacy of traditional Chinese medicine, which binds to proteins, are potential targets for asthma therapy. Another approach is a combination therapy, when herbal medicines used together with modern medicines enhance asthma outcomes as compared to the use of pharmacotherapies alone. ${ }^{87}$ Zhou et al ${ }^{88}$ indicated that acupuncture ameliorates asthmatic pulmonary function associated to metallothionein-2. Based on all the above studies, it is hypothesised that the combination of acupuncture with TMPs treatments also provide better therapeutic outcomes. Future research on asthma treatment should be focused in this direction and more advanced studies are required to confirm the use of TMPs in combination with conventional therapies.

\section{Conclusion and Future Perspectives}

In this review, we highlighted 27 traditional medicinal plant species and two polyherbal extracts that have been investigated for their anti-asthmatic potential using OVA-induced models. Our review revealed that most of the investigated medicinal plants and their extracts promote attenuation of asthma symptoms in experimental animals and supports its traditional information. Nevertheless, the toxicological profiles of the plant extracts remain unreported since most of the studies focused only on their anti-asthmatic potentials. Additionally, there is a severe lack of information on 


\begin{tabular}{|c|c|c|c|c|c|c|c|c|}
\hline 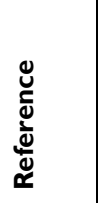 & 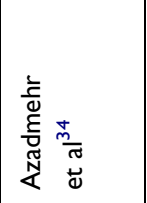 & 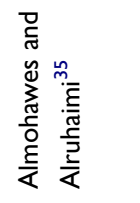 & 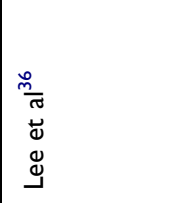 & 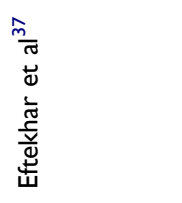 & 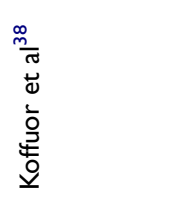 & 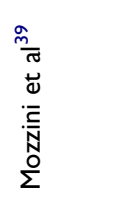 & 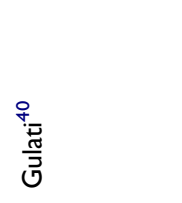 & 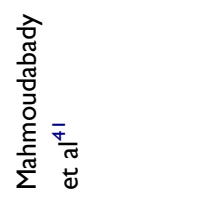 \\
\hline 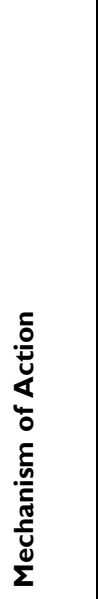 & 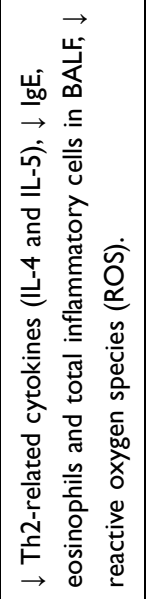 & 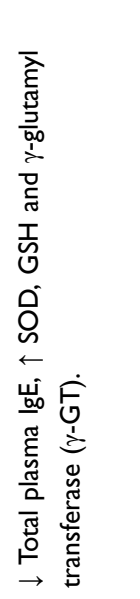 & 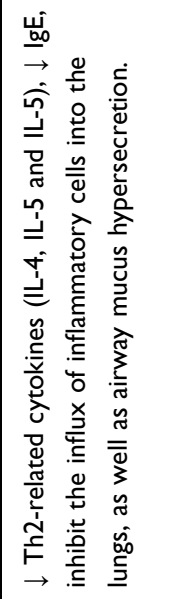 & 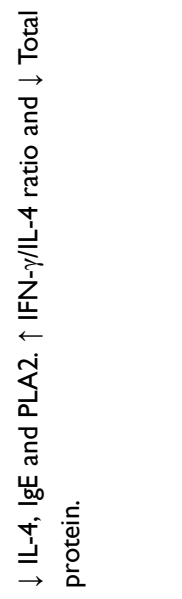 & 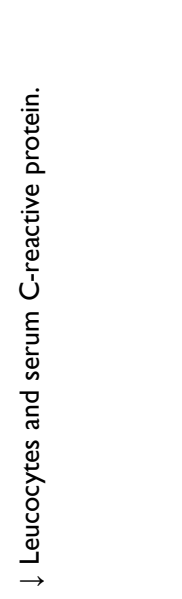 & 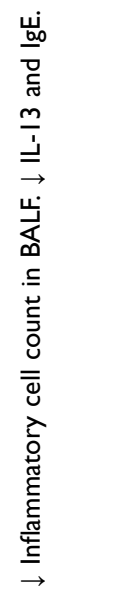 & 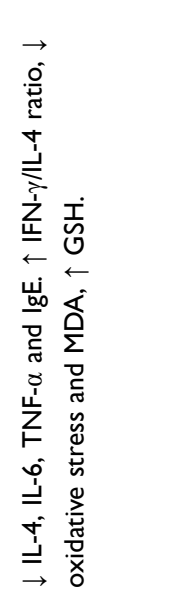 & 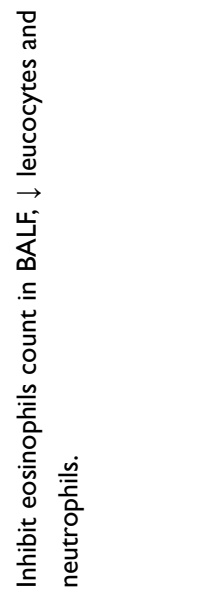 \\
\hline 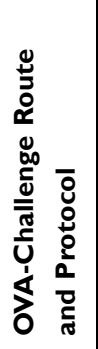 & 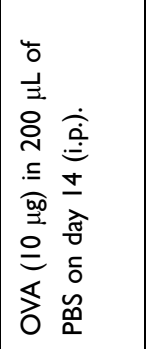 & 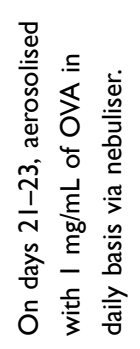 & 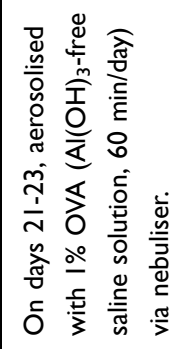 & 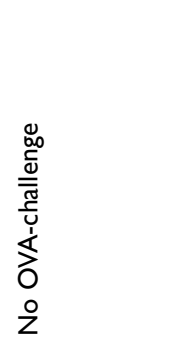 & 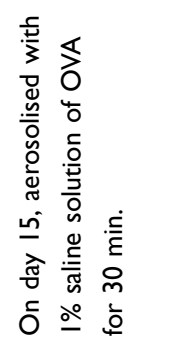 & 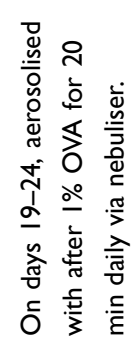 & 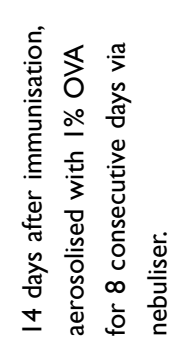 & 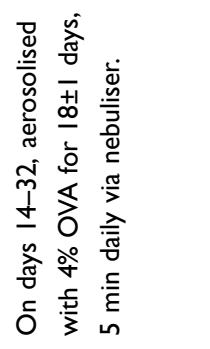 \\
\hline 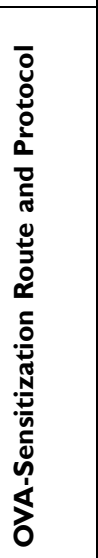 & 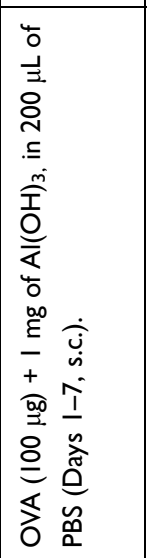 & 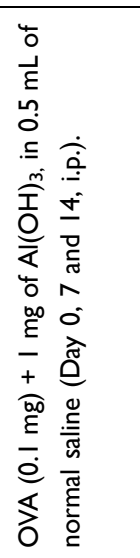 & 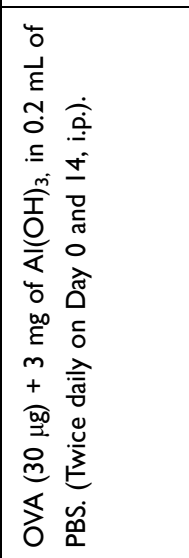 & 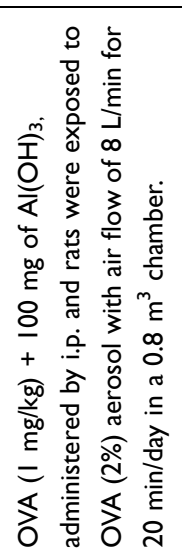 & 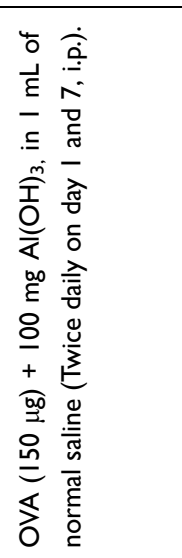 & 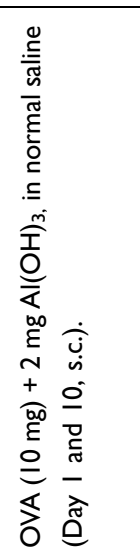 & 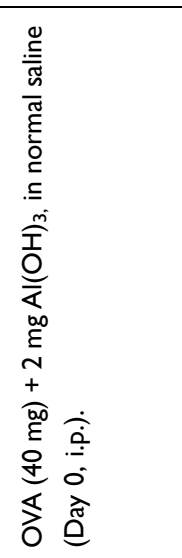 & 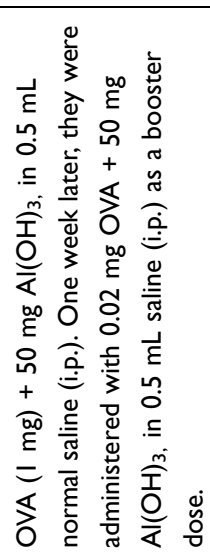 \\
\hline 施 & 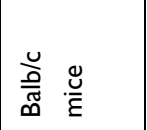 & 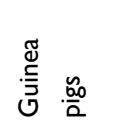 & 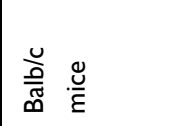 & 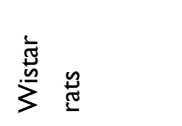 & 离 & 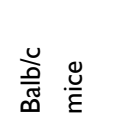 & 鴶 & 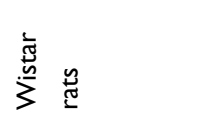 \\
\hline 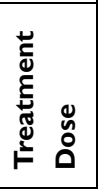 & 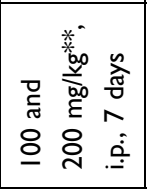 & 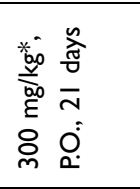 & 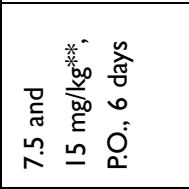 & 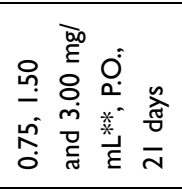 & 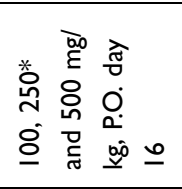 & 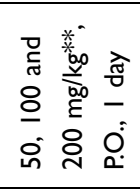 & 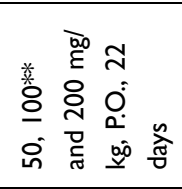 & 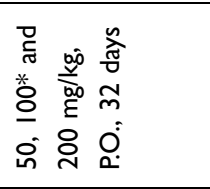 \\
\hline 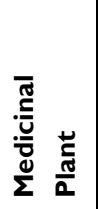 & 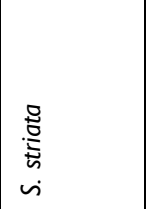 & 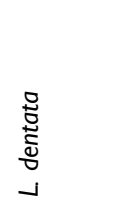 & 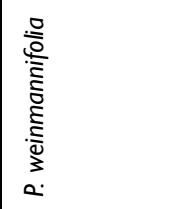 & 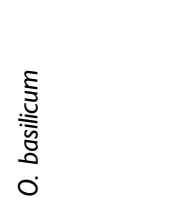 & 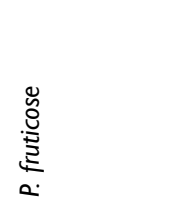 & $\begin{array}{l}\mathscr{D} \\
\stackrel{\Xi}{\Xi} \\
I \\
I\end{array}$ & 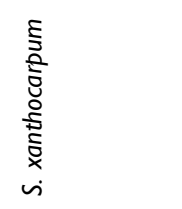 & ن \\
\hline
\end{tabular}




\begin{tabular}{|c|c|c|c|c|c|c|c|}
\hline 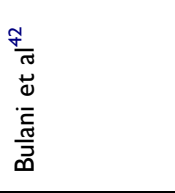 & 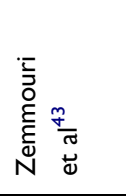 & 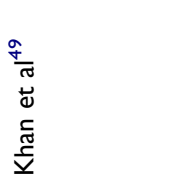 & $\begin{array}{l}\frac{q}{\pi} \\
\frac{2}{0} \\
\overline{0} \\
\frac{\partial}{U}\end{array}$ & 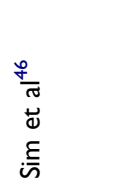 & 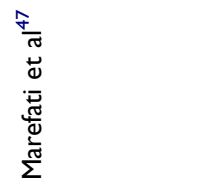 & 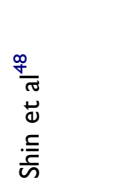 & 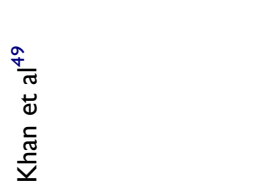 \\
\hline 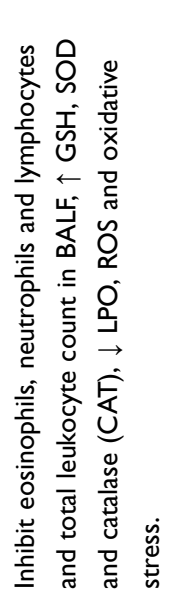 & 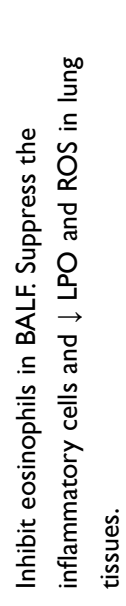 & 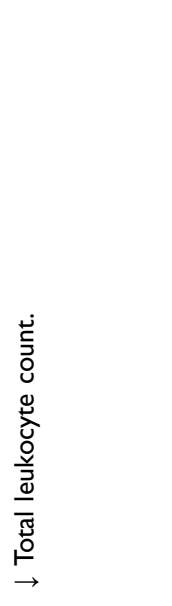 & 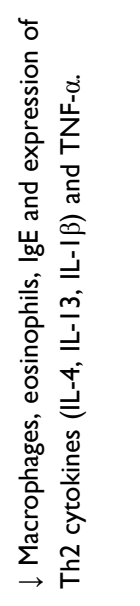 & 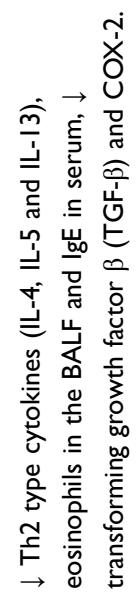 & 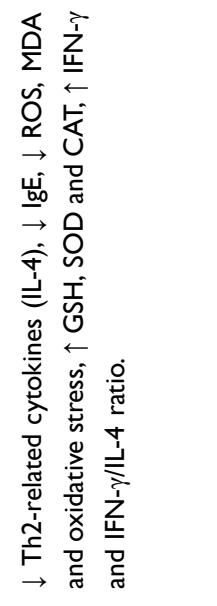 & 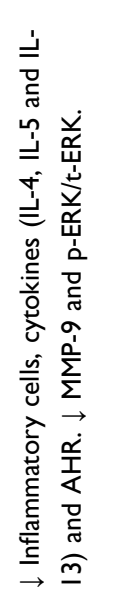 & 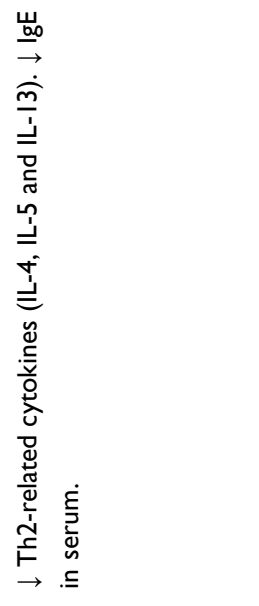 \\
\hline 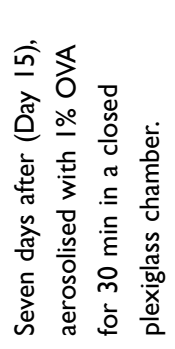 & 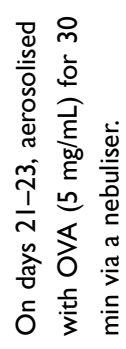 & 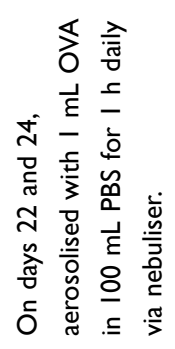 & 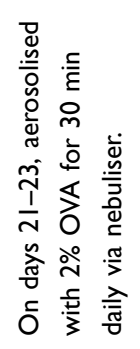 & 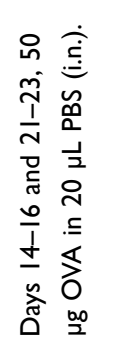 & 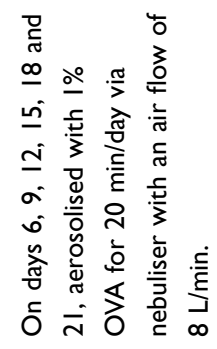 & 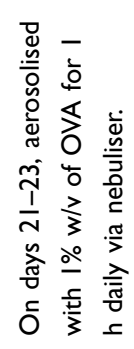 & 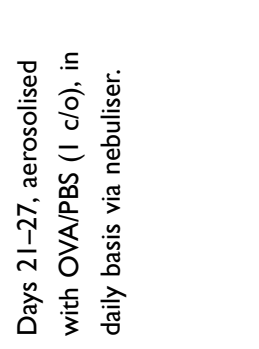 \\
\hline 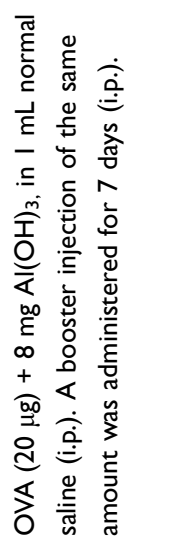 & 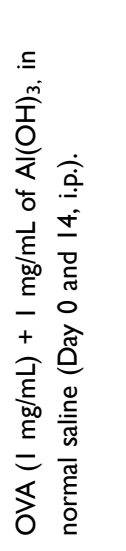 & 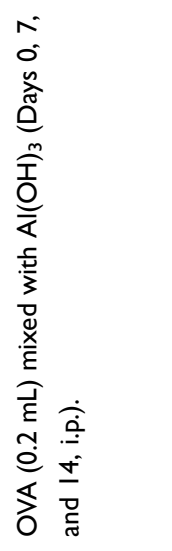 & 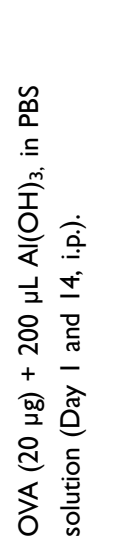 & 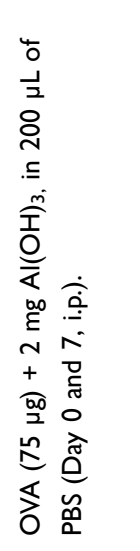 & 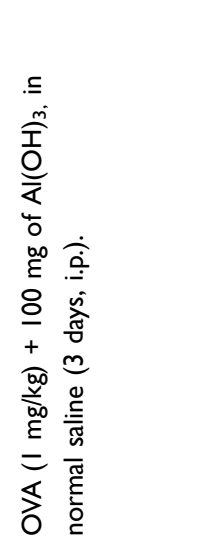 & 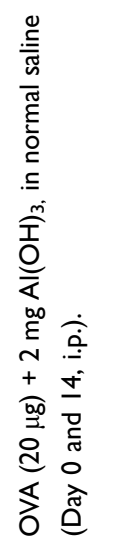 & 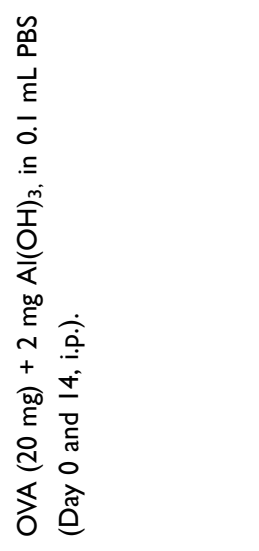 \\
\hline 总 & 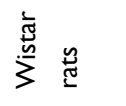 & 莺 & 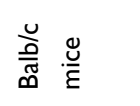 & 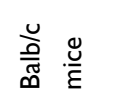 & 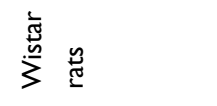 & 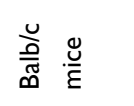 & 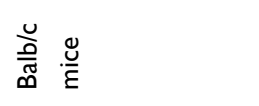 \\
\hline 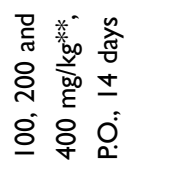 & 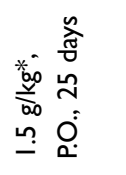 & 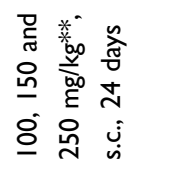 & 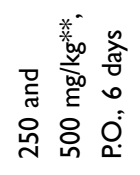 & 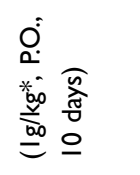 & 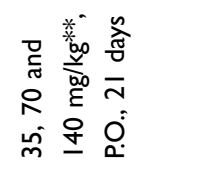 & 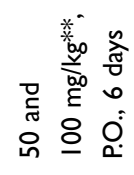 & 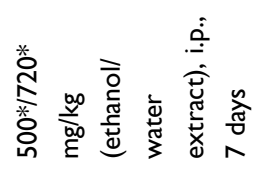 \\
\hline 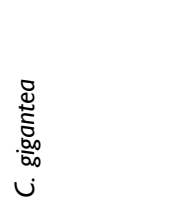 & 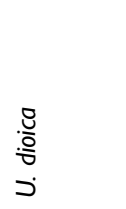 & 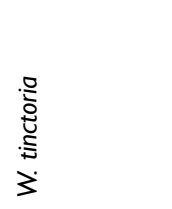 & 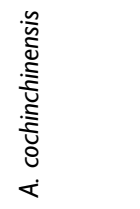 & 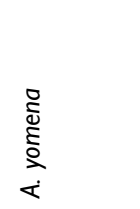 & $\begin{array}{l}0 \\
\stackrel{8}{0} \\
\dot{<} \\
\dot{\alpha}\end{array}$ & 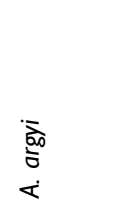 & 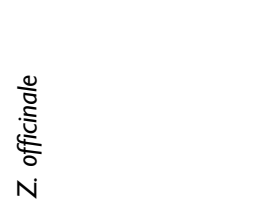 \\
\hline
\end{tabular}




\begin{tabular}{|c|c|c|c|c|c|c|c|}
\hline 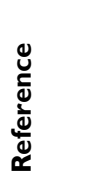 & 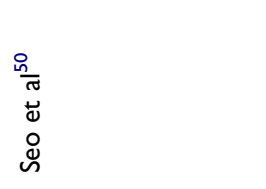 & $\begin{array}{l}\overline{\frac{n}{n}} \\
\stackrel{5}{0} \\
\stackrel{0}{0} \\
\stackrel{0}{0}\end{array}$ & 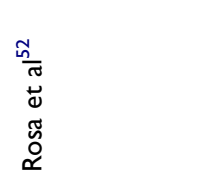 & 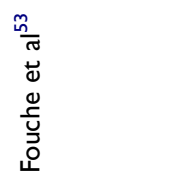 & 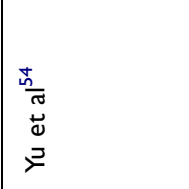 & 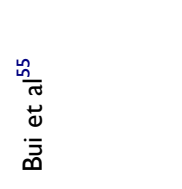 & 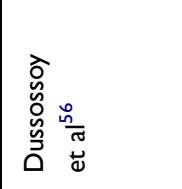 \\
\hline 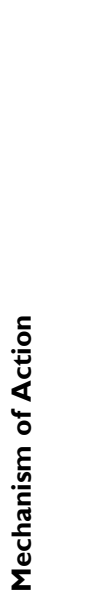 & 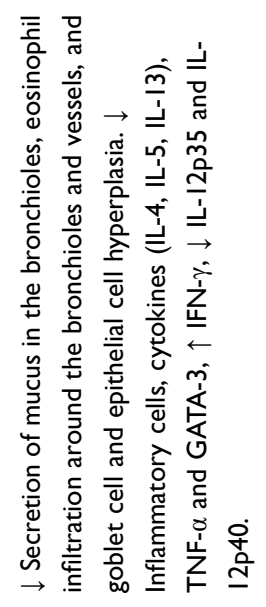 & 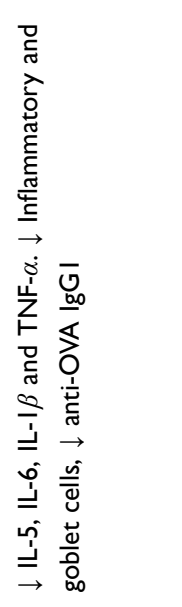 & 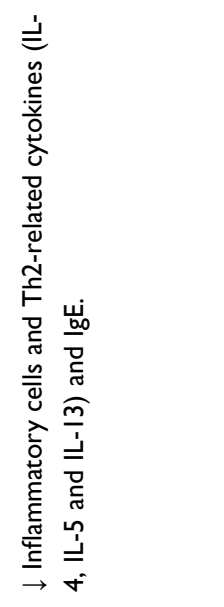 & 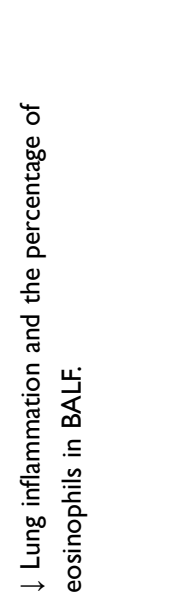 & 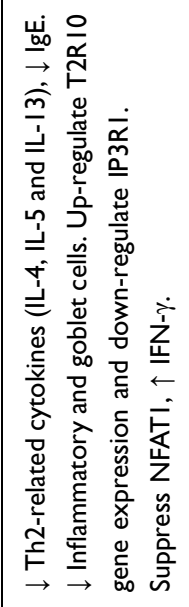 & 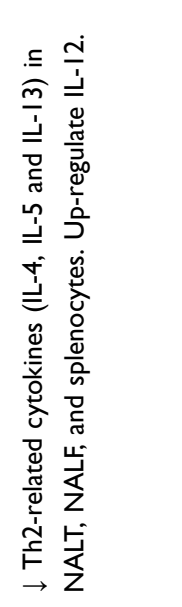 & 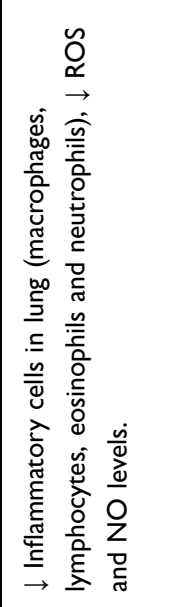 \\
\hline 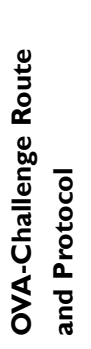 & 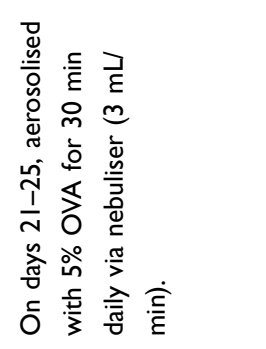 & 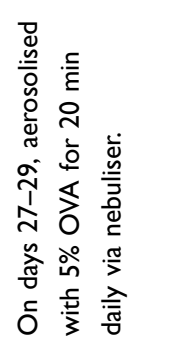 & 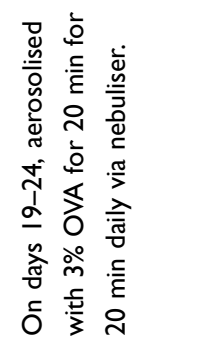 & 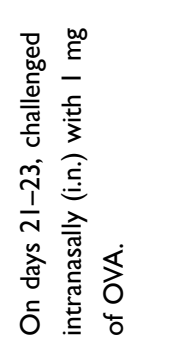 & 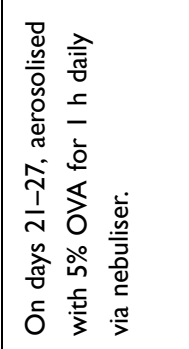 & 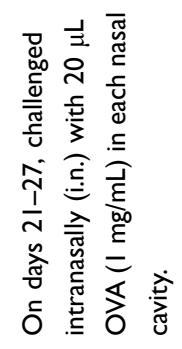 & 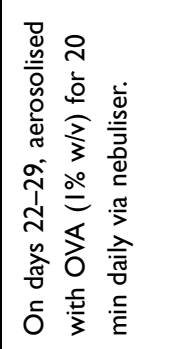 \\
\hline 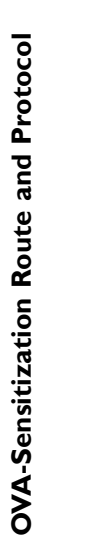 & 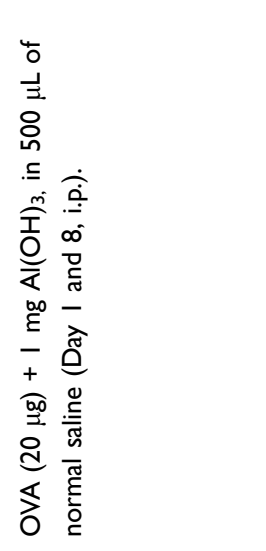 & 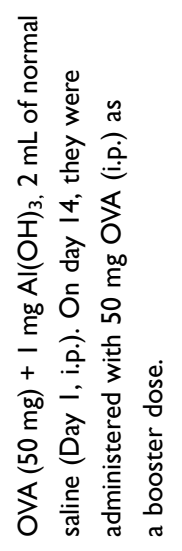 & 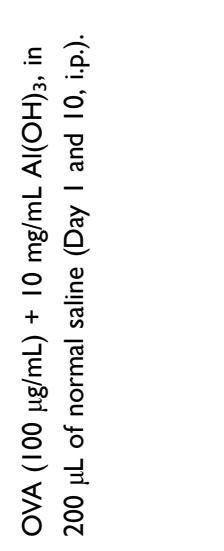 & 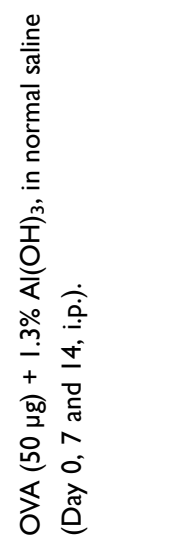 & 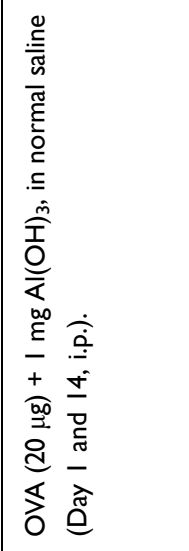 & 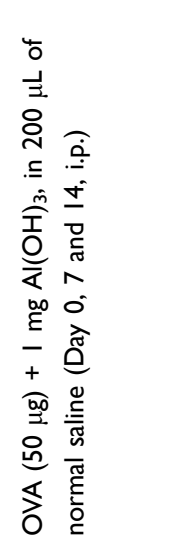 & 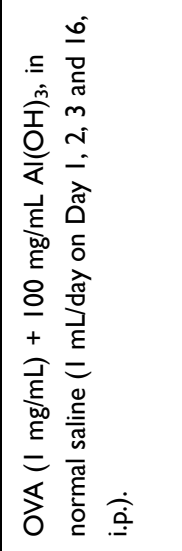 \\
\hline 赔 & 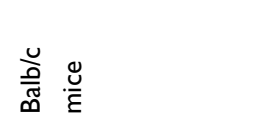 & 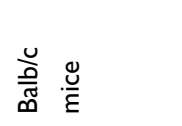 & 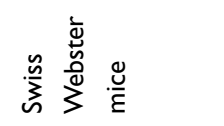 & 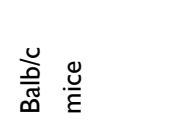 & 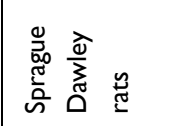 & 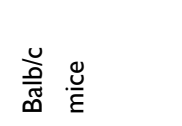 & 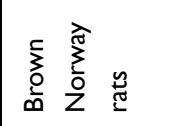 \\
\hline 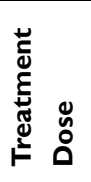 & 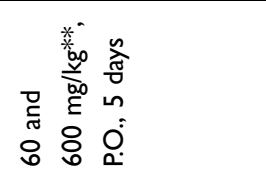 & 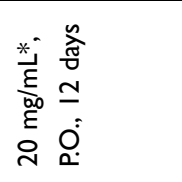 & 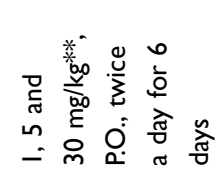 & 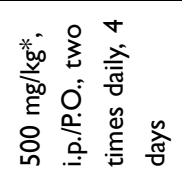 & 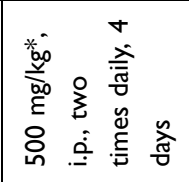 & 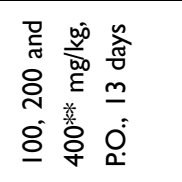 & 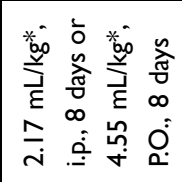 \\
\hline 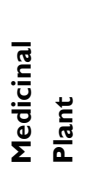 & 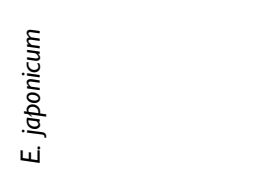 & 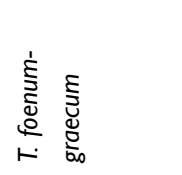 & 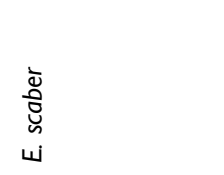 & 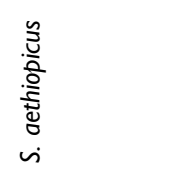 & $\begin{array}{l}\text { Z } \\
: 5 \\
: 5 \\
5 \\
i\end{array}$ & 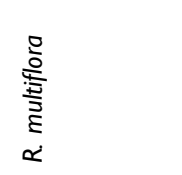 & $\begin{array}{l}\stackrel{.0}{\overline{0}} \\
\text { 妾 } \\
\dot{\Sigma}\end{array}$ \\
\hline
\end{tabular}




\begin{tabular}{|c|c|c|c|c|c|c|}
\hline 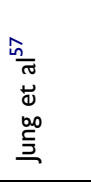 & 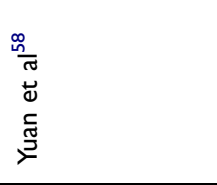 & 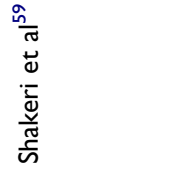 & 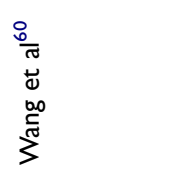 & & 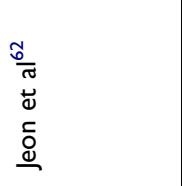 & 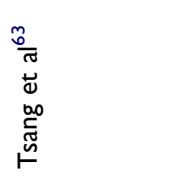 \\
\hline 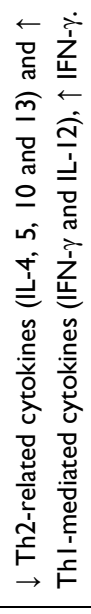 & 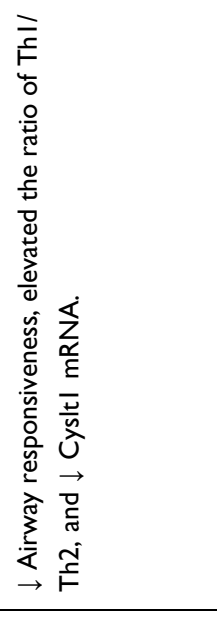 & 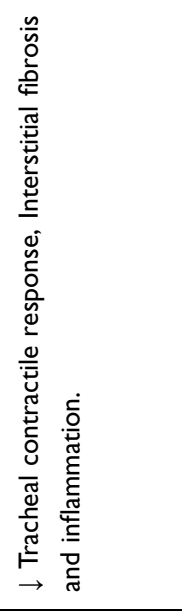 & 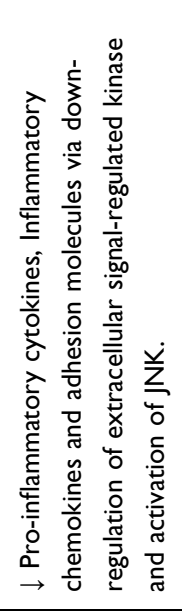 & & 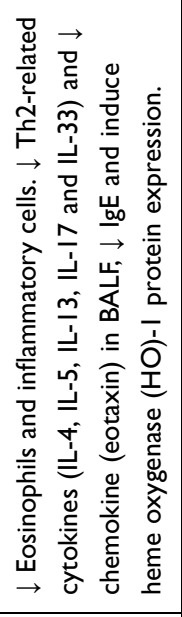 & 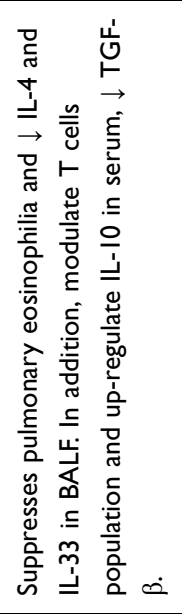 \\
\hline 1 & 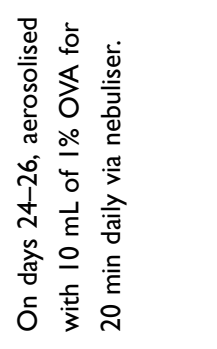 & 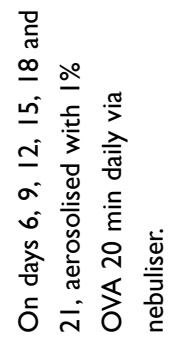 & 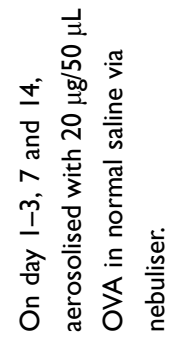 & & 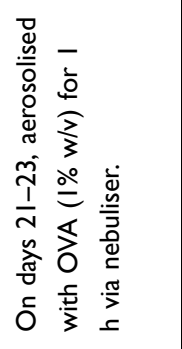 & 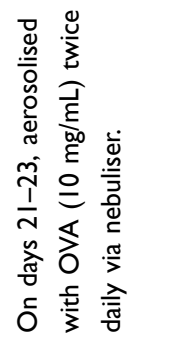 \\
\hline 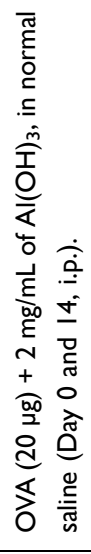 & 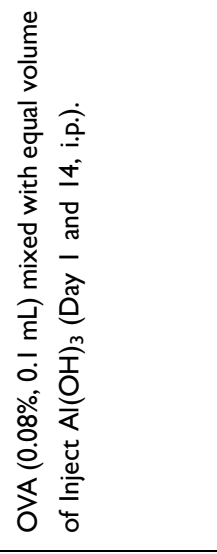 & 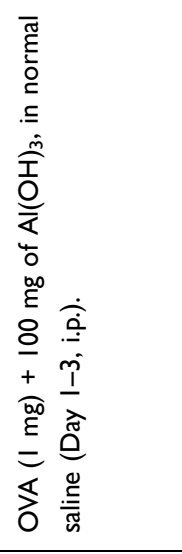 & 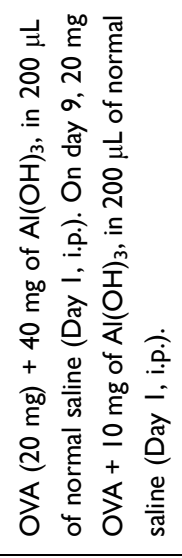 & & 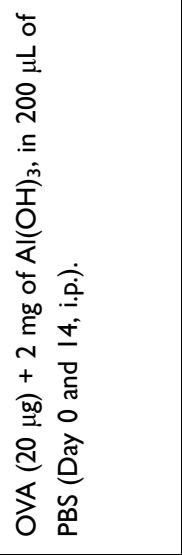 & 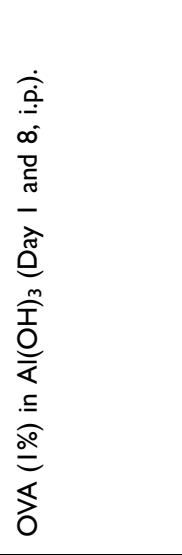 \\
\hline 峁 & 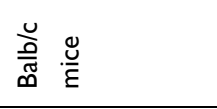 & 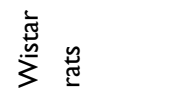 & $\stackrel{\stackrel{\circ}{\circ}}{\frac{\mathscr{O}}{\tilde{\omega}}}$ & & 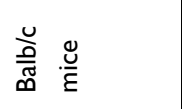 & 怨 \\
\hline 1 & 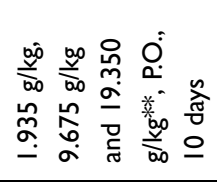 & 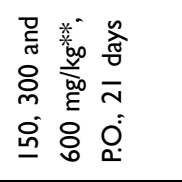 & 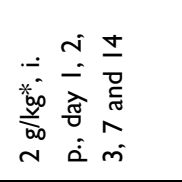 & & 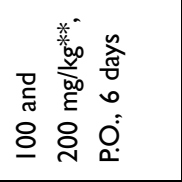 & 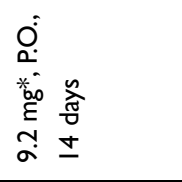 \\
\hline 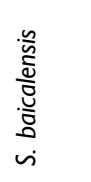 & 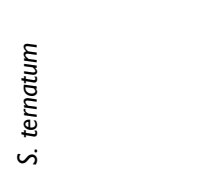 & $\begin{array}{l}\text { 品 } \\
\stackrel{\delta}{\cup}\end{array}$ & 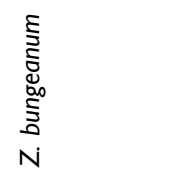 & 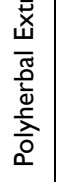 & 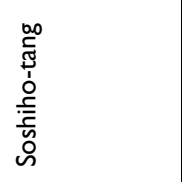 & 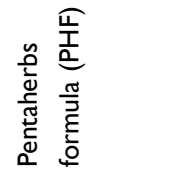 \\
\hline
\end{tabular}




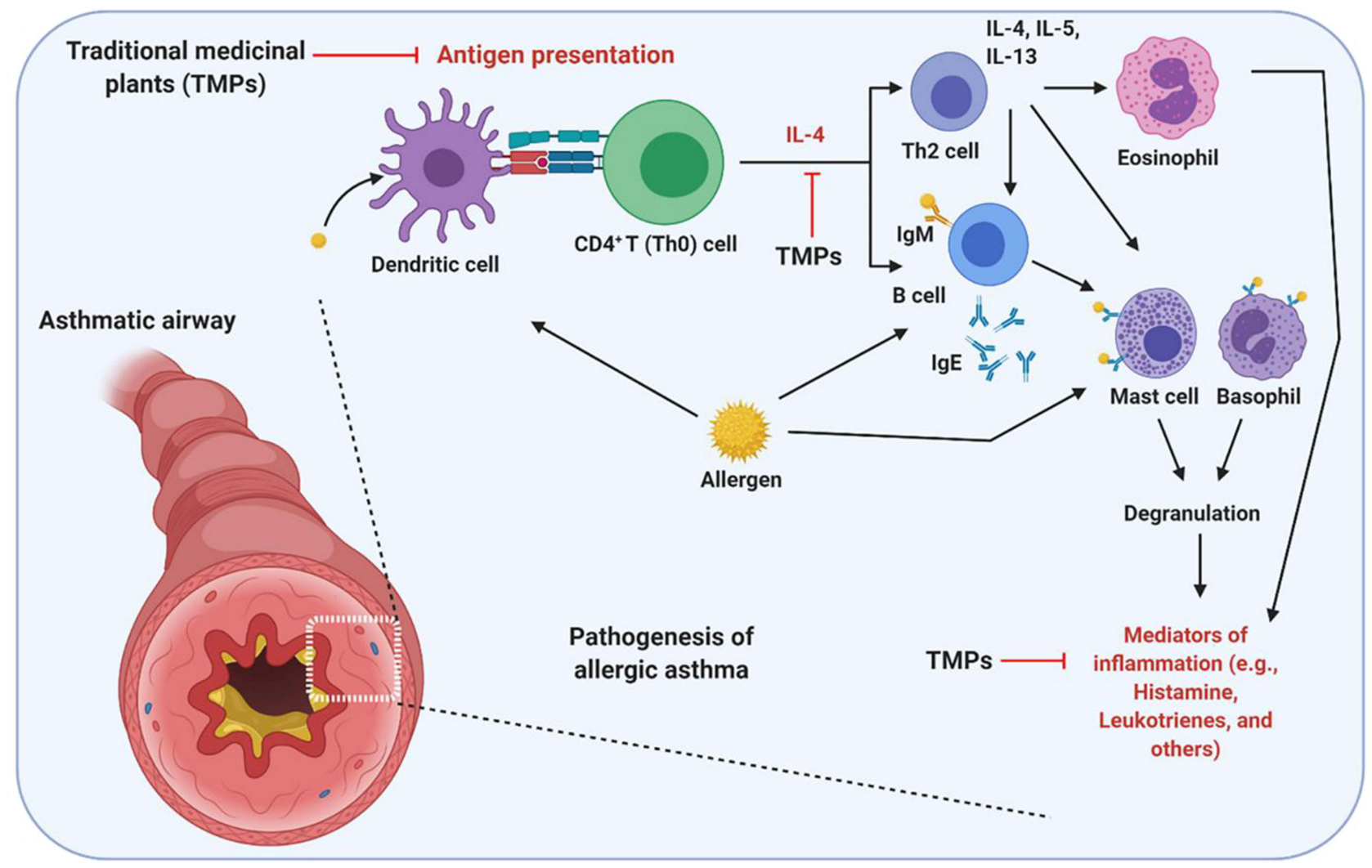

Figure 3 Possible mechanism of action of TMPs against OVA-induced asthma. As an allergen, OVA-induces airway inflammation and hyperresponsiveness. As a protein OVA recognized, processed and presented by the antigen-presenting cells. Upon presentation, CD4 ${ }^{+} \mathrm{T}$ helper (Th0) cells are activated and polarized toward Th2 phenotype, which activates eosinophils (Eosinophilic asthma), mast cells, and basophils to secret inflammatory mediators. OVA directly or indirectly (via Th cells mediated) activates the B lymphocytes to secret IgE, which is the main correlative factor in allergen-induced asthma. TMPs may either act by inhibiting antigen presentation or cytokine secretion or other inflammatory mediators (histamine, leukotrienes, and others). The figure was created with the support of https://biorender.com under the paid subscription.

the efficacy, safety and the required dosage to induce the in vivo anti-asthma activity which should be the focus in future studies. The lack of scientific reports and limited information on the isolated compounds responsible for anti-asthmatic actions is another negative factor that should be addressed in future studies. In conclusion, the vast potentials of the TMPs should be explored for the development of new drugs to enhance existing antiasthmatic treatment as an adjuvant or to be used as a new armamentarium against asthma.

\section{Consent for Publication}

The final version of the manuscript was reviewed by all the authors and consented to its submission.

\section{Acknowledgments}

The authors would like to thank Universiti Kuala Lumpur Royal College of Medicine Perak, Malaysia for providing necessary facilities and resources to complete this study for publication.

\section{Author Contributions}

S.A., and M.S. conceived the idea. S.A., M.S., and S.R. B. designed, collected the literature, interpreted the data, analyzed the data, drafted and revised the manuscript. All the authors have made noteworthy contributions to the study design, data collection, review and interpretation; have engaged in the drafting or revision of the article; have agreed to submit to the current journal; have given final approval of the version to be published; and have agreed to be responsible for all aspects of the work.

\section{Disclosure}

The authors have no conflict of interest associated with this work or publication. There was no significant financial support for this work.

\section{References}

1. Global Initiative for Asthma. Global Strategy for Asthma Management and Prevention. Vol. 2020. Global Initiative for Asthma; 2016.

2. Kaufman G. Asthma: pathophysiology, diagnosis and management. Nurs Stand. 2011;26(5):48. doi:10.7748/ns.26.5.48.s55 
3. Pedersen SE, Hurd SS, Lemanske JRF, et al. Global strategy for the diagnosis and management of asthma in children 5 years and younger. Pediatr Pulmonol. 2011;46(1):1-17. doi:10.1002/ ppul.21321

4. Mohammed S, Goodacre S. Intravenous and nebulised magnesium sulphate for acute asthma: systematic review and meta-analysis. Emerg Med. 2007;24(12):823-830.

5. Adams BK, Cydulka RK. Asthma evaluation and management. Emerg Med Clin N Am. 2003;21(2):315-330. doi:10.1016/S07338627(03)00015-4

6. Schaneberg BT, Crockett S, Bedir E, et al. The role of chemical fingerprinting: application to Ephedra. Phytochemistry. 2003;62 (6):911-918. doi:10.1016/S0031-9422(02)00716-1

7. Barnes PJ. Drugs for asthma. Br J Pharmacol. 2006;147(S1): S297-S303. doi:10.1038/sj.bjp.0706437

8. Barnes PJ. The cytokine network in asthma and chronic obstructive pulmonary disease. J Clin Invest. 2008;118(11):3546-3556. doi:10.1172/JCI36130

9. Lugnier C. Cyclic nucleotide phosphodiesterase (PDE) superfamily: a new target for the development of specific therapeutic agents. Pharmacol Ther. 2006;109(3):366-398.

10. DiMartino SJ. Idiopathic inflammatory myopathy: treatment options. Curr Rheumatol Rep. 2008;10(4):321. doi:10.1007/ s11926-008-0051-4

11. Kesler SM, Sprenkle MD, David WS, et al. Severe weakness complicating status asthmaticus despite minimal duration of neuromuscular paralysis. Intensive Care Medicine. 2009;35 (1):157-160. doi:10.1007/s00134-008-1267-5

12. Che C-T, George V, Ijinu $\mathrm{T}$, et al. Traditional Medicine. Pharmacognosy: Elsevier; 2017:15-30.

13. Albuquerque UPD, Hanazaki N. As pesquisas etnodirigidas na descoberta de novos fármacos de interesse médico e farmacêutico: fragilidades e pespectivas. Revista Brasileira De Farmacognosia. 2006;16:678-689. doi:10.1590/S0102-695X2006000500015

14. Yuan G, Wahlqvist ML, He G, et al. Natural products and anti-inflammatory activity.. Asia Pac J Clin Nutr. 2006;15 (2):143-152.

15. Bonam SR, Wu YS, Tunki L, et al. What has come out from phytomedicines and herbal edibles for the treatment of cancer? Chem Med Chem. 2018;13(18):1854-1872. doi:10.1002/ cmdc. 201800343

16. Yuan H, Ma Q, Ye L, et al. The traditional medicine and modern medicine from natural products. Molecules. 2016;21(5):559. doi:10.3390/molecules21050559

17. Fabricant DS, Farnsworth NR. The value of plants used in traditional medicine for drug discovery.. Environ Health Perspect. 2001;109 Suppl 1(suppl 1):69-75. doi:10.1289/ ehp.01109s169

18. Alves RR, Rosa IM. Biodiversity, traditional medicine and public health: where do they meet? Journal of Ethnobiology and Ethnomedicine. 2007;3(1):14. doi:10.1186/1746-4269-3-14

19. Bonam SR, Partidos CD, Halmuthur SKM, et al. An overview of novel adjuvants designed for improving vaccine efficacy. Trends Pharmacol Sci. 2017;38(9):771-793. doi:10.1016/j.tips.2017.06.002

20. Chen C, Sun N, Li Y, et al. A BALB/c mouse model for assessing the potential allergenicity of proteins: comparison of allergen dose, sensitization frequency, timepoint and sex. Food Chem Toxicol. 2013;62:41-47. doi:10.1016/j.fct.2013.08.004

21. Shin YS, Takeda K, Gelfand EW. Understanding asthma using animal models. Allergy Asthma Immunol Res. 2009;1(1):10-18. doi:10.4168/aair.2009.1.1.10

22. Schülke S, Albrecht M. Mouse models for food allergies: where do we stand? Cells. 2019;8(6):546. doi:10.3390/cells8060546

23. Yu Q-L, Chen Z. Establishment of different experimental asthma models in mice.. Exp Ther Med. 2018;15(3):2492-2498. doi:10.3892/etm.2018.5721
24. Chen C, Lianhua L, Nana S, et al. Development of a BALB/c mouse model for food allergy: comparison of allergy-related responses to peanut agglutinin, $\beta$-lactoglobulin and potato acid phosphatase. Toxicol Res. 2017;6(2):251-261. doi:10.1039/ C6TX00371K

25. Aun MV, Bonamichi-Santos R, Arantes-Costa FM, et al. Animal models of asthma: utility and limitations. J Asthma Allergy. 2017;10:293. doi:10.2147/JAA.S121092

26. Fehrenbach $\mathrm{H}$, Wagner $\mathrm{C}$, Wegmann M. Airway remodeling in asthma: what really matters. Cell Tissue Res. 2017;367 (3):551-569. doi:10.1007/s00441-016-2566-8

27. Lee M-F, Yang K-J, Wang NM, et al. The development of a murine model for Forcipomyia taiwana (biting midge) allergy. PLoS One. 2014;9(3):e91871. doi:10.1371/journal.pone.0091871

28. Hogan SP, Matthaei KI, Young JM, et al. A novel T cell-regulated mechanism modulating allergen-induced airways hyperreactivity in BALB/c mice independently of IL-4 and IL-5.. J Immunol. 1998;161(3):1501-1509.

29. Zhou C, Ludmila T, Sun N, et al. BALB/c mice can be used to evaluate allergenicity of different food protein extracts. Food Agric Immunol. 2016;27(5):589-603. doi:10.1080/09540105.2015.1129600

30. Bodinier M, Leroy M, Ah-Leung S, et al. Sensitization and elicitation of an allergic reaction to wheat gliadins in mice. J Agric Food Chem. 2009;57(4):1219-1225. doi:10.1021/jf8 $02898 \mathrm{u}$

31. Morafo V, Srivastava K, Huang C-K, et al. Genetic susceptibility to food allergy is linked to differential TH2-TH1 responses in $\mathrm{C} 3 \mathrm{H} / \mathrm{HeJ}$ and BALB/c mice. J Allergy Clin Immunol. 2003;111 (5):1122-1128. doi:10.1067/mai.2003.1463

32. Hylkema M, Hoekstra M, Luinge M, et al. The strength of the OVA-induced airway inflammation in rats is strain dependent. Clin Exp Immunol. 2002;129(3):390-396. doi:10.1046/j.13652249.2002.01938.x

33. Simpson JL, Scott R, Boyle MJ, et al. Inflammatory subtypes in asthma: assessment and identification using induced sputum. Respirology. 2006;11(1):54-61. doi:10.1111/j.1440-1843.2006.00 784.x

34. Azadmehr A, Hajiaghaee R, Zohal MA, et al. Protective effects of Scrophularia striata in Ovalbumin-induced mice asthma model. DARU J Pharmaceutical Sci. 2013;21(1):56-62. doi:10.1186/ 2008-2231-21-56

35. Almohawes Z, Alruhaimi H. Effect of Lavandula dentata extract on Ovalbumin-induced Asthma in Male Guinea Pigs. Brazilian Journal of Biology. 2020;80(1):87-96. doi:10.1590/1519-6984.191485

36. Lee J-W, Min J-H, Kim M-G, et al. Pistacia weinmannifolia root exerts a protective role in ovalbumin-induced lung inflammation in a mouse allergic asthma model.. Int J Mol Med. 2019;44 (6):2171-2180. doi:10.3892/ijmm.2019.4367

37. Eftekhar N, Moghimi A, Roshan NM, et al. Immunomodulatory and anti-inflammatory effects of hydro-ethanolic extract of Ocimum basilicum leaves and its effect on lung pathological changes in an ovalbumin-induced rat model of asthma. BMC Complement Altern Med. 2019;19(1):349. doi:10.1186/s12906019-2765-4

38. Koffuor GA, Boye A, Ofori-Amoah J, et al. Anti-inflammatory and safety assessment of Polyscias fruticosa (L.) Harms (Araliaceae) leaf extract in ovalbumin-induced asthma. J Phytopharm. 2014;3(5):337-342.

39. Mozzini MT, Ferrera Costa H, Carvalho Vieira G, et al. Antiasthmatic and anxiolytic effects of Herissantia tiubae, a Brazilian medicinal plant. Immun Inflamm Dis. 2016;4(2):201-212. doi:10.1002/iid3.107

40. Gulati K. Evaluation of Anti-Inflammatory and Immunomodulatory Effects of Aqueous Extract of Solanum Xanthocarpum in Experimental Models of Bronchial Asthma. EC Pharmacol Toxicol. 2016;2:241-250. 
41. Mahmoudabady M, Neamati A, Vosooghi S, et al. Hydroalcoholic extract of Crocus sativus effects on bronchial inflammatory cells in ovalbumin sensitized rats. Avicenna J Phytomed. 2013;3 (4):356.

42. Bulani V, Biyani K, Kale R, et al. Inhibitory effect of Calotropis gigantea extract on ovalbumin-induced airway inflammation and Arachidonic acid induced inflammation in a murine model of asthma. Int J Cur Bio Med Sci. 2011;1(2):19-25.

43. Zemmouri H, Sekiou O, Ammar S, et al. Urtica dioica attenuates ovalbumin-induced inflammation and lipid peroxidation of lung tissues in rat asthma model. Pharm Biol. 2017;55(1):1561-1568. doi:10.1080/13880209.2017.1310905

44. Khan Z, Ansari I. Antiasthmatic activity of the ethanol extract of leaves of Wrightia tinctoria.. Asian J Pharm Clin Res. 2018;11 (9):136-139. doi:10.22159/ajpcr.2018.v11i9.26151

45. Choi JY, Kim J, Park JJ, et al. The anti-inflammatory effects of fermented herbal roots of Asparagus cochinchinensis in an ovalbumin-induced asthma model. J Clin Med. 2018;7(10):377. doi: $10.3390 /$ jcm 7100377

46. Sim JH, Lee HS, Lee S, et al. Anti-Asthmatic Activities of an Ethanol Extract of Aster yomena in an Ovalbumin-Induced Murine Asthma Model. J Med Food. 2014;17(5):606-611. doi:10.1089/jmf.2013.2939

47. Marefati N, Eftekhar N, Kaveh M, et al. The effect of Allium cepa extract on lung oxidant, antioxidant, and immunological biomarkers in ovalbumin-sensitized rats. Med Princ Pract. 2018;27 (2):122-128. doi:10.1159/000487885

48. Shin N-R, Ryu H-W, Ko J-W, et al. Artemisia argyi attenuates airway inflammation in ovalbumin-induced asthmatic animals. J Ethnopharmacol. 2017;209:108-115. doi:10.1016/j.jep.2017.07. 033

49. Khan AM, Shahzad M, Raza Asim M, et al. Zingiber officinale ameliorates allergic asthma via suppression of Th2-mediated immune response. Pharm Biol. 2015;53(3):359-367. doi:10.31 09/13880209.2014.920396

50. Seo J-H, Bang M, Kim G, et al. Erythronium japonicum attenuates histopathological lung abnormalities in a mouse model of ovalbumin-induced asthma. Int $J$ Mol Med. 2016;37 (5):1221-1228. doi:10.3892/ijmm.2016.2541

51. Piao $\mathrm{CH}$, Bui TT, Song $\mathrm{CH}$, et al. Trigonella foenum-graecum alleviates airway inflammation of allergic asthma in ovalbumin-induced mouse model. Biochem Biophys Res Commun. 2017;482(4):1284-1288. doi:10.1016/j.bbrc.2016.12. 029

52. Rosa SIG, Rios-Santos F, Balogun SO, et al. Hydroethanolic extract from Echinodorus scaber Rataj leaves inhibits inflammation in ovalbumin-induced allergic asthma. J Ethnopharmacol. 2017;203:191-199. doi:10.1016/j.jep.2017.03.025

53. Fouche $\mathrm{G}$, Nieuwenhuizen $\mathrm{N}$, Maharaj V, et al. Investigation of in vitro and in vivo anti-asthmatic properties of Siphonochilus aethiopicus. $J$ Ethnopharmacol. 2011;133(2):843-849. doi:10.1016/j.jep.2010.11.014

54. Yu B, Cai W, Zhang -H-H, et al. Selaginella uncinata flavonoids ameliorated ovalbumin-induced airway inflammation in a rat model of asthma. $J$ Ethnopharmacol. 2017;195:71-80. doi:10.1016/j.jep.2016.11.049

55. Bui TT, Kwon D-A, Choi DW, et al. Rosae multiflorae fructus extract and its four active components alleviate ovalbumin-induced allergic inflammatory responses via regulation of Th1/Th2 imbalance in BALB/c rhinitis mice. Phytomedicine. 2019;55:238-248. doi:10.1016/j.phymed.2018. 06.044

56. Dussossoy E, Bichon F, Bony E, et al. Pulmonary anti-inflammatory effects and spasmolytic properties of Costa Rican noni juice (Morinda citrifolia L.). J Ethnopharmacol. 2016;192:264-272. doi:10.1016/j.jep.2016.07.038
57. Jung SY, Lee S-Y, Choi DW, et al. Skullcap (Scutellaria baicalensis) hexane fraction inhibits the permeation of ovalbumin and regulates Th1/2 immune responses. Nutrients. 2017;9(11):1184. doi:10.3390/nu9111184

58. Yuan Y, Yang B, Ye Z, et al. Sceptridium ternatum extract exerts antiasthmatic effects by regulating Th1/Th2 balance and the expression levels of leukotriene receptors in a mouse asthma model. J Ethnopharmacol. 2013;149(3):701-706. doi:10.1016/j. jep.2013.07.032

59. Shakeri F, Roshan NM, Boskabady MH. Hydro-ethanolic extract of Curcuma longa affects tracheal responsiveness and lung pathology in ovalbumin-sensitized rats. Int $J$ Vitamin Nutrition Res. 2020;90(1-2):141-150. doi:10.1024/0300-9831/ a000524

60. Wang J-Q, Li X-W, Liu M, et al. Inhibitory effect of Zanthoxylum bungeanum seed oil on ovalbumin-induced lung inflammation in a murine model of asthma. Mol Med Rep. 2016;13(5):4289-4302. doi:10.3892/mmr.2016.5050

61. Ohtake N, Nakai Y, Yamamoto M, et al. The herbal medicine Shosaiko-to exerts different modulating effects on lung local immune responses among mouse strains. Int Immunopharmacol. 2002;2(2-3):357-366. doi:10.1016/ S1567-5769(01)00161-8

62. Jeon W-Y, Shin H-K, Shin I-S, et al. Soshiho-tang water extract inhibits ovalbumin-induced airway inflammation via the regulation of heme oxygenase-1. BMC Complement Altern Med. 2015;15(1):1-10. doi:10.1186/s12906-015-0857-3

63. Tsang MS, Jiao D, Chan BC, et al. Anti-inflammatory activities of pentaherbs formula, berberine, gallic acid and chlorogenic acid in atopic dermatitis-like skin inflammation. Molecules. 2016;21 (4):519. doi:10.3390/molecules21040519

64. Tamri P. A mini-review on phytochemistry and pharmacological activities of Scrophularia striata. J Herbmed Pharmacol. 2019;8 (2):85-89. doi:10.15171/jhp.2019.14

65. Ullah R, Alqahtani AS, Noman OMA, et al. A review on ethno-medicinal plants used in traditional medicine in the Kingdom of Saudi Arabia. Saudi J Biol Sci. 2020;27(10):2706. doi:10.1016/j.sjbs.2020.06.020

66. Dash G, Mohanty KKGR, Sahoo D, et al. Traditional medicinal plants used for the treatment of asthma in Bhubaneswar, Odisha. Int J Herb Med. 2018;6(5):57-60.

67. Javadi B, Sahebkar A, Emami SA. Medicinal plants for the treatment of asthma: a traditional Persian medicine perspective. Curr Pharm Des. 2017;23(11):1623-1632. doi:10.2174/ 1381612822666161021143332

68. Savithramma N, Sulochana C, Rao K. Ethnobotanical survey of plants used to treat asthma in Andhra Pradesh, India. J Ethnopharmacol. 2007;113(1):54-61. doi:10.1016/j.jep.2007.04.004

69. Younis A, Younis W, Asif H, et al. Traditional medicinal plants used for respiratory disorders in Pakistan: a review of the ethno-medicinal and pharmacological evidence. Chinese Medicine. 2018;13(1):48. doi:10.1186/s13020-018-0204-y

70. Dulla O, Jahan FI. Ethnopharmacological survey on traditional medicinal plants at Kalaroa Upazila, Satkhira District, Khulna Division, Bangladesh. J Intercult Ethnopharmacol. 2017;6 (3):316. doi:10.5455/jice.20170719010256

71. Napagoda MT, Sundarapperuma T, Fonseka D, et al. An ethnobotanical study of the medicinal plants used as anti-inflammatory remedies in Gampaha District, Western Province, Sri Lanka. Scientifica. 2018;2018:1-8. doi:10.1155/ 2018/9395052

72. Panthong A, Kanjanapothi D, Taylor W. Ethnobotanical review of medicinal plants from Thai traditional books, Part I: plants with anti-inflammatory, anti-asthmatic and antihypertensive properties. J Ethnopharmacol. 1986;18(3):213-228. doi:10.1016/0378-8741(86) 90001-2 
73. Gbekley HE, Katawa G, Karou SD, et al. Ethnobotanical study of plants used to treat asthma in the maritime region in Togo. Afr $J$ Tradit Complement Altern Med. 2016;14(1):196-212. doi:10.21010/ajtcam.v14i1.22

74. Mali RG, Dhake AS. A review on herbal antiasthmatics. Orient Pharm Exp Med. 2011;11(2):77-90. doi:10.1007/s13596-0110019-1

75. Singh BB, Khorsan R, Vinjamury SP, et al. Herbal treatments of asthma: a systematic review. J Asthma. 2007;44(9):685-698. doi:10.1080/02770900701247202

76. Maema LP, Potgieter M, Mahlo SM. Invasive alien plant species used for the treatment of various diseases in Limpopo Province, South Africa. Afr J Tradit Complement Altern Med. 2016;13 (4):223-231. doi:10.21010/ajtcam.v13i4.29

77. Kaladhar D. Traditional and Ayurvedic medicinal plants from India: practices and treatment for human diseases. LAP. 2012;176.

78. Eisenbrand G, Tang W. Handbook of Chinese Medicinal Plants: Chemistry, Pharmacology, Toxicology. John Wiley \& Sons; 2010.

79. Wiart C. Medicinal Plants of China, Korea, and Japan: Bioresources for Tomorrow's Drugs and Cosmetics. CRC press; 2012.

80. Khare C. World Healing Plants for Tomorrow. Daya Publishing House; 2018.

81. Kim H, Song M-J. Traditional plant-based therapies for respiratory diseases found in North Jeolla Province, Korea. J Altern Complement Med. 2012;18(3):287-293. doi:10.1089/ acm.2010.0848

82. Thakur VR, Khuman V, Beladiya JV, et al. An experimental model of asthma in rats using ovalbumin and lipopolysaccharide allergens. Heliyon. 2019;5(11):e02864. doi:10.1016/j.heliyon.2019.e02864

83. Zhao S, Jiang Y, Yang X, et al. Lipopolysaccharides promote a shift from Th2-derived airway eosinophilic inflammation to Th17-derived neutrophilic inflammation in an ovalbumin-sensitized murine asthma model. J Asthma. 2017;54 (5):447-455. doi:10.1080/02770903.2016.1223687

84. Lowe A, Thomas RS, Nials A, et al. LPS exacerbates functional and inflammatory responses to ovalbumin and decreases sensitivity to inhaled fluticasone propionate in a guinea pig model of asthma. Br J Pharmacol. 2015;172(10):2588-2603. doi:10.1111/ bph. 13080

85. Clyne A, Yang AWH, Li M, et al. Traditional medicines for asthma in children and adults: a systematic review of placebo-controlled studies. Int J Clin Pract. 2019;73(12): e13433. doi:10.1111/ijcp.13433

86. Wang Y, Chen Y-J, Xiang C, et al. Discovery of potential asthma targets based on the clinical efficacy of Traditional Chinese Medicine formulas. $J$ Ethnopharmacol. 2020;252:112635. doi:10.1016/j.jep.2020.112635

87. Shergis JL, Wu L, Zhang AL, et al. Herbal medicine for adults with asthma: a systematic review. J Asthma. 2016;53(6):650-659. doi:10.3109/02770903.2015.1101473

88. Zhou -D-D, Ran J, Li -C-C, et al. Metallothionein-2 is associated with the amelioration of asthmatic pulmonary function by acupuncture through protein phosphorylation. Biomed Pharmacother. 2020;123:109785. doi:10.1016/j.biopha.2019.109785

89. Zhao X, Sun H, Hou A, et al. Antioxidant properties of two gallotannins isolated from the leaves of Pistacia weinmannifolia. Biochimica Et Biophysica Acta (BBA) - General Subjects. 2005;1725(1):103-110. doi:10.1016/j.bbagen.2005.04.015

90. Chen S, Wu X, Ji Y, et al. Isolation and characterization of microsatellite loci in Pistacia weinmannifolia (Anacardiaceae). Int J Mol Sci. 2011;12(11):7818-7823. doi:10.3390/ijms12117818

91. Ci X, Chu X, Chen C, et al. Oxytetracycline attenuates allergic airway inflammation in mice via inhibition of the NF- $\kappa \mathrm{B}$ pathway. J Clin Immunol. 2011;31(2):216-227. doi:10.1007/s10875010-9481-7
92. Javanmardi J, Khalighi A, Kashi A, et al. Chemical Characterization of Basil (Ocimum basilicum L.) Found in Local Accessions and Used in Traditional Medicines in Iran. J Agric Food Chem. 2002;50(21):5878-5883. doi:10.1021/ jf020487q

93. Prakash P, Gupta N. Therapeutic uses of Ocimum sanctum Linn (Tulsi) with a note on eugenol and its pharmacological actions: a short review.. Indian J Physiol Pharmacol. 2005;49(2):125.

94. Do T. Vietnamese Medicinal Plants and Remedies. Hanoi. Medicine Publisher; 2000:828-830.

95. Asumeng Koffuor G, Boye A, Kyei S, et al. Anti-asthmatic property and possible mode of activity of an ethanol leaf extract of Polyscias fruticosa. Pharm Biol. 2016;54(8):1354-1363. doi: $10.3109 / 13880209.2015 .1077465$

96. Hanh TTH, Dang NH, Dat NT. $\alpha$-Amylase and $\alpha$-Glucosidase Inhibitory Saponins from Polyscias fruticosa Leaves. J Chem. 2016;2016:1-5. doi:10.1155/2016/2082946

97. Falcão-Silva VS, Silva DA, Souza V, et al. Modulation of drug resistance in staphylococcus aureus by a kaempferol glycoside from Herissantia tiubae (Malvaceae). Phytother Res. 2009;23 (10):1367-1370. doi:10.1002/ptr.2695

98. Ghani A. Medicinal Plants of Bangladesh: Chemical Constituents and Uses. Vol. 2. Asiatic society of Bangladesh; 1998:81.

99. Parmar S, Gangwal A, Sheth N. Solanum xanthocarpum (yellow berried night shade): a review. Der Pharm Lett. 2010;2 (4):373-383.

100. Rios J, Recio M, Giner R, et al. An update review of saffron and its active constituents. Phytother Res. 1996;10(3):189-193. doi:10.1002/(SICI)1099-1573(199605)10:3<189::AIDPTR754>3.0.CO;2-C

101. Maheshwari P, Singh U. Dictionary of Economic Plants in India. Dictionary of Economic Plants in India. New Delhi: Indian Council of Agricultural Research; 1965:38-39.

102. Warrier P, Nambiar V, Ramankutty C, et al. Indian Medicinal Plants. New Delhi: Orient Longman; 1995:341-343.

103. Kirtikar K, Basu B. Indian medicinal plants. Phytother Res. 1935;1607-1609.

104. Randall C, Randall H, Dobbs F, et al. Randomized controlled trial of nettle sting for treatment of base-of-thumb pain. J R Soc Med. 2000;93(6):305-309. doi:10.1177/014107680009300607

105. Chrubasik S, Enderlein W, Bauer R, et al. Evidence for antirheumatic effectiveness of Herba Urticae dioicae in acute arthritis: a pilot study. Phytomedicine. 1997;4(2):105-108. doi:10.1016/ S0944-7113(97)80052-9

106. Mittman P. Randomized, Double-Blind Study of Freeze-Dried Urtica dioica in the Treatment of Allergic Rhinitis. Planta Med. 1990;56(01):44-47. doi:10.1055/s-2006-960881

107. Srivastava R. A review on phytochemical, pharmacological, and pharmacognostical profile of Wrightia tinctoria: adulterant of kurchi. Pharmacogn Rev. 2014;8(15):36. doi:10.4103/09737847.125528

108. Xiong D, Yu L-X, Yan X, et al. Effects of Root and Stem Extracts of Asparagus cochinchinensis on Biochemical Indicators Related to Aging in the Brain and Liver of Mice. Am J Chin Med. 2011;39 (04):719-726. doi:10.1142/S0192415X11009159

109. Roldán E, Sanchez-Moreno C, de Ancos B, et al. Characterisation of onion (Allium cepa L.) by-products as food ingredients with antioxidant and antibrowning properties. Food Chem. 2008;108 (3):907-916. doi:10.1016/j.foodchem.2007.11.058

110. Lee BK, Jung Y-S. Allium cepa extract and quercetin protect neuronal cells from oxidative stress via PKC- $\varepsilon$ inactivation/ ERK1/2 activation. Oxid Med Cell Longev. 2016;2016:1-9.

111. Kim AR, Ko HJ, Chowdhury MA, et al. Chemical constituents on the aerial parts of Artemisia selengensis and their IL-6 inhibitory activity. Arch Pharm Res. 2015;38(6):1059-1065. doi:10.1007/ s12272-014-0543-x 
112. Kim JK, Shin E-C, Lim H-J, et al. Characterization of nutritional composition, antioxidative capacity, and sensory attributes of Seomae mugwort, a native Korean variety of Artemisia argyi $\mathrm{H}$. J Anal Methods Chem. 2015;1-6.

113. Yun C, Jung Y, Chun W, et al. Anti-Inflammatory Effects of Artemisia Leaf Extract in Mice with Contact Dermatitis In Vitro and In Vivo. Mediators Inflamm. 2016;2016:1-8. doi:10.1155/ 2016/8027537

114. Lee NY, Chung K-S, Jin JS, et al. Effect of chicoric acid on mast cell-mediated allergic inflammation in vitro and in vivo. $J$ Nat Prod. 2015;78(12):2956-2962. doi:10.1021/acs.jnatprod.5b00668

115. Yao X, Wu D, Dong N, et al. Moracin C, A Phenolic Compound Isolated from Artocarpus heterophyllus, Suppresses Lipopolysaccharide-Activated Inflammatory Responses in Murine Raw264.7 Macrophages. Int J Mol Sci. 2016;17 (8):1199. doi:10.3390/ijms17081199

116. Ahui MLB, Champy P, Ramadan A, et al. Ginger prevents Th2-mediated immune responses in a mouse model of airway inflammation. Int Immunopharmacol. 2008;8(12):1626-1632. doi:10.1016/j.intimp.2008.07.009

117. Townsend EA, Siviski ME, Zhang Y, et al. Effects of ginger and its constituents on airway smooth muscle relaxation and calcium regulation. Am J Respir Cell Mol Biol. 2013;48(2):157-163. doi: $10.1165 / \mathrm{rcmb} .2012-02310 \mathrm{OC}$

118. Shin YJ, Jung DY, Ha HK, et al. Anticancer effect of Erythronium japonicum extract on ICR mouse and L1210 cells with alteration of antioxidant enzyme activities. Korean J Food Sci Technol. 2004;36:968-973.

119. Khalil MI, Ibrahim MM, El-Gaaly GA, et al. Trigonella foenum (Fenugreek) induced apoptosis in hepatocellular carcinoma cell line, HepG2, mediated by upregulation of p53 and proliferating cell nuclear antigen. Biomed Res Int. 2015;2015.

120. Bieski IGC, Rios Santos F, De Oliveira RM, et al. Ethnopharmacology of medicinal plants of the Pantanal region (Mato Grosso, Brazil). Evid Based Complement Altern Med. 2012;2012:1-36. doi:10.1155/2012/272749

121. De la Cruz MG. Plantas Medicinais De Mato Grosso: A Farmacopéia Popular Dos Raizeiros. Plantas Medicinais De Mato Grosso: A Farmacopéia Popular Dos Raizeiros. Brazil: Carlini Caniato; 2008:224.

122. Messias MCTB, Menegatto M, Prado ACC, et al. Uso popular de plantas medicinais e perfil socioeconômico dos usuários: um estudo em área urbana em Ouro Preto, MG, Brasil. Revista Brasileira De Plantas Medicinais. 2015;17(1):76-104. doi:10.1590/1983-084X/12_139

123. Hutchings A. Zulu Medicinal Plants: An Inventory. Pietermaritzburg: University of Natal press; 1996:64.

124. Steenkamp V. Traditional herbal remedies used by South African women for gynaecological complaints. $J$ Ethnopharmacol. 2003;86(1):97-108. doi:10.1016/S0378-8741(03)00053-9
125. Steenkamp V, Grimmer H, Semano M, et al. Antioxidant and genotoxic properties of South African herbal extracts. Mutat Res Genet Toxicol Environ Mutagen. 2005;581(1-2):35-42. doi:10.1016/j.mrgentox.2004.10.009

126. Zou H, Yi M-L, Xu K-P, et al. Two new flavonoids from Selaginella uncinata. $J$ Asian Nat Prod Res. 2016;18 (3):248-252. doi:10.1080/10286020.2015.1063617

127. Zheng J-X, Zheng Y, Zhi H, et al. New 3', $8^{\prime \prime}$-Linked Biflavonoids from Selaginella uncinata Displaying Protective Effect against Anoxia. Molecules. 2011;16(8):6206-6214. doi:10.3390/ molecules 16086206

128. Chen K-L, Li J, Lei X. Comparison of cytotoxic activities of extracts from Selaginella species. Pharmacogn Mag. 2014;10 (40):529. doi:10.4103/0973-1296.141794

129. Cheng BCY, Yu H, Su T, et al. A herbal formula comprising Rosae Multiflorae Fructus and Lonicerae Japonicae Flos inhibits the production of inflammatory mediators and the IRAK-1/TAK1 and TBK1/IRF3 pathways in RAW 264.7 and THP-1 cells. $J$ Ethnopharmacol. 2015;174:195-199. doi:10.1016/j.jep.2015. 08.018

130. Whistler WA. Tongan Herbal Medicine. Isle Botanica, Honolulu, Hawaii: University of Hawaii Press; 1992:89-90.

131. Li-Weber M. New therapeutic aspects of flavones: the anticancer properties of Scutellaria and its main active constituents Wogonin, Baicalein and Baicalin. Cancer Treat Rev. 2009;35 (1):57-68. doi:10.1016/j.ctrv.2008.09.005

132. Zhang Z, Lian X, Li S, et al. Characterization of chemical ingredients and anticonvulsant activity of American skullcap (Scutellaria lateriflora). Phytomedicine. 2009;16(5):485-493. doi:10.1016/j.phymed.2008.07.011

133. Wang S, Ruan J, Zhuang J. Small Chunhua oral mouse model of chronic bronchitis and pathological forms of influence and expectorant effects. Fujian Chin Med. 2001;32:18-19.

134. Omosa L, Midiwo J, Kuete V. Curcuma Longa. Medicinal Spices and Vegetables from Africa. Elsevier; 2017:425-435.

135. Zhang M, Wang J, Zhu L, et al. Zanthoxylum bungeanum Maxim. (Rutaceae): a Systematic Review of Its Traditional Uses, Botany, Phytochemistry, Pharmacology, Pharmacokinetics, and Toxicology. Int J Mol Sci. 2017;18(10):2172. doi:10.3390/ ijms18102172

136. Rahman M, Alimuzzaman M, Ahmad S, et al. Antinociceptive and antidiarrhoeal activity of Zanthoxylum rhetsa. Fitoterapia. 2002;73(4):340-342. doi:10.1016/S0367-326X(02)00083-7

137. Larché M, Akdis CA, Valenta R. Immunological mechanisms of allergen-specific immunotherapy. Nat Rev Immunol. 2006;6 (10):761-771. doi:10.1038/nri1934

\section{Publish your work in this journal}

The Journal of Asthma and Allergy is an international, peer-reviewed open-access journal publishing original research, reports, editorials and commentaries on the following topics: Asthma; Pulmonary physiology; Asthma related clinical health; Clinical immunology and the immunological basis of disease; Pharmacological interventions and new therapies. The manuscript management system is completely online and includes a very quick and fair peer-review system, which is all easy to use. Visit http://www.dovepress.com/testimonials.php to read real quotes from published authors. 